\title{
Coordinated Optical and Radar measurements of Low Velocity Meteors
}

\author{
Peter Brown ${ }^{\mathrm{a}, \mathrm{b}}$, Robert J. Weryk ${ }^{\mathrm{c}}$ \\ ${ }^{a}$ Department of Physics and Astronomy, University of Western Ontario, London, Ontario, N6A 3K7, \\ Canada \\ ${ }^{b}$ Western Institute for Earth and Space Exploration, University of Western Ontario, London, Ontario, N6A \\ 5B7, Canada \\ ${ }^{c}$ Institute for Astronomy, University of Hawaii, Honolulu HI, 96822, USA
}

\begin{abstract}
To better estimate which luminous efficiency $(\tau)$ value is compatible with contemporary values of the ionization coefficient $(\beta)$, we report a series of simultaneous optical and specular echo radar measurements of low speed $(\mathrm{v}<20 \mathrm{~km} / \mathrm{s})$ meteors. We focus on the low speed population as secondary ionization is not relevant and the initial trail radii are small, minimizing model assumptions required to estimate electron line density. By using the large decrease in expected ionization coefficient at such low speeds, we attempt to better define the likely ratio of photon to electron production. This provides an estimate of the probable luminous efficiency, given that recent lab measurements of ionization efficiency agree with established theory (Jones, 1997; DeLuca et al., 2018) suggesting $\beta$ is more constrained than $\tau$.

Optical measurements were performed with two pairs of autonomously operated electron-multiplied charge coupled device cameras (EMCCDs) co-located with the multi-frequency Canadian Meteor Orbit Radar (CMOR) (Brown et al., 2008). Using the timing and geometry of individual meteors measured by both the radar and multi-station EMCCD systems, the portion of the optical lightcurve corresponding to each specular radar echo is measured and the received echo power used to estimate an electron line density. A total of 1249 simultaneous EMCCD and radar meteors were identified from observations between 2017 - 2019 with 55 having in atmosphere speeds
\end{abstract}

Email address: pbrown@uwo.ca (Peter Brown)

Preprint submitted to Elsevier

July 9, 2020 
below $20 \mathrm{~km} / \mathrm{s}$. A subset of 36 events were analyzed in detail, with 29 having speed $<20 \mathrm{~km} / \mathrm{s}$. These meteors had G-band magnitudes at the specular radar point between +4 and +7.7 , with an average radiant power of $5 \mathrm{~W}$ (assuming a $945 \mathrm{~W}$ power for a zero magnitude meteor). These correspond to a typical magnitude of +6 . Following the procedure in Weryk \& Brown (2013b), the ratio of electron line density (q) to radiant power (I) provides a direct estimate of the ionization coefficient $(\beta)$ to luminous efficiency $(\tau)$ ratio for each event. We find that $\beta / \tau$ strongly correlates with radiant power. All our simultaneous meteors had asteroidal-like orbits and six were found to be probable iron meteoroids, representing $20 \%$ of our slow $<20 \mathrm{~km} / \mathrm{s}$ sample. Luminous efficiency values averaged $0.6 \%$ at low speed, ranging from $<0.1 \%$ to almost $30 \%$. No trend of luminous efficiency with speed was apparent, though a weak correlation between higher values of $\tau$ and radiant power may be present.

\section{Introduction}

The measurement of meteoroid mass using either optical or radar observations of meteors requires knowledge of the amount of ablation energy partitioned into photon or electron production. The associated luminous efficiency $(\tau)$ and ionization coefficient $(\beta)$ are quantities which have historically been measured in the laboratory (eg. Slattery \& Friichtenicht (1967); Friichtenicht et al. (1968)) or estimated from meteor measurements. While the range in luminous efficiency estimates is very large (Subasinghe et al. 2017), recent laboratory measurements of the ionization efficiency (DeLuca et al. 2018, Thomas et al. 2016) are in comparatively better agreement with the theoretical estimates from Jones (1997). Moreover, both measurements and theory suggest a rapid drop in ionization efficiency for speeds below $20 \mathrm{~km} / \mathrm{s}$.

Characterizing and understanding the meteoroid population encountering the Earth at low velocities ( $v<20 \mathrm{~km} / \mathrm{s}$ ) has become increasingly important in recent years. In the last decade, new dynamical meteoroid models (eg. (Nesvorný et al., 2010, Yang \& Ishiguro, 2015) ) have predicted a large population of small, slow meteoroids originating from Jupiter-family comets which should dominate the mass influx to the Earth (Carrillo-Sánchez et al., 2016), in contrast to predictions from some earlier models 
(Liou et al., 1995). These new models have produced estimates for the speed distribution as a function of meteoroid mass for such models (Carrillo-Sánchez et al. 2016), providing testable predictions. Similarly, recent studies (eg. Borovička et al. (2005); Campbell-Brown (2015) ) have suggested that a significant fraction of low speed meteoroids may be iron in composition, with the fraction appearing to increase with decreasing mass (Capek et al. 2019).

Interpreting the physics behind the preceding examples depend on accurate estimation of meteoroid mass. This is a difficult problem, as meteoroid mass is always indirectly inferred from observations necessarily interpreted through the lens of model assumptions. At low speed, meteoroid mass becomes particularly uncertain as both the luminous efficiency (the fraction of total meteoroid kinetic energy which becomes radiation, $\tau$ ) and the ionization coefficient (the number of electrons produced per ablated atom, $\beta$ ) change at speeds below $20 \mathrm{~km} / \mathrm{s}$ (Jones, 1997; Jones \& Halliday, 2001: Weryk \& Brown 2013b, DeLuca et al., 2018). Dynamical mass estimates are complicated by the ubiquitous presence of fragmentation (Subasinghe et al., 2016) which is significant at even very small meteoroid sizes (Mathews et al., 2010).

One approach to improve the accuracy of meteoroid mass estimates is to observe common meteor events using simultaneous (but independent) techniques. Comparing common mass estimates from different techniques allows for a sense of the global mass accuracy for individual meteors. In some cases, it is possible to combine measurements across techniques to better estimate energy conversion efficiencies. This approach has been most commonly employed through the fusion of optical and radar measurements of meteors, eg. Campbell-Brown et al. (2012), through optical and infrasound (Silber et al. 2015) as well as optical and LIDAR (Klekociuk et al., 2005), which have been combined to produce independent mass estimates.

Past studies using optical and radar measurements have focused either on comparing radar head echo (radial scattering) measurements to optical records (Nishimura et al. 2001) or radar specular (transverse scattering) returns to optical signatures (Weryk \& Brown, 2012). The limitation of the former is that inference of meteoroid mass from radar head echo power returns depends on detailed knowledge of the plasma distribution in the immediate vicinity of the ablating meteoroid (Marshall et al., 2017), 
requiring models with many poorly constrained parameters (Close et al. 2004).

Specular radar returns, in contrast, are limited to probing the average electron line density in the vicinity of the first fresnel zone (Ceplecha et al., 1998) and therefore can provide a snapshot only of mass loss in a short segment ( $1-2 \mathrm{~km}$ ) of trail. Furthermore, inference of the electron line density from transverse scattering depends on knowledge of the radial electron distribution (Kaiser \& Closs, 1952), creating similar complications in interpretation as for the head echo case. However, for long wavelengths or low ablation heights, the initial trail radius may be small compared to the radar wavelength, minimizing the effects of attenuation (Jones \& Campbell-Brown, 2005). In this limit, the reflected power is a relatively slowly varying function of the electron line density (particularly for echoes in the underdense regime), implying that the resulting electron-line density estimates are comparatively robust to modelling choices (Weryk \& Brown 2013b). This also implies that estimates of electron line density are most accurate for lower altitude (and lower electron line density) echoes, a feature we exploit in this work.

The present study is an extension of the earlier work described in Weryk \& Brown (2012) and Weryk \& Brown (2013b). That investigation focused on directly measuring the ratio of $\frac{\beta}{\tau}$ through comparison of specular echoes detected by the Canadian Meteor Orbit Radar (CMOR) and co-located (but manually operated) image intensified CCD cameras. Through adoption of a probable model for $\beta$, the corresponding values for $\tau$ were estimated. The major difference between this earlier work and the current study is the extension to much fainter optical meteors through the use of Electron-Multiplied Charge Coupled Devices (EMCCDs) as well as a focus on low speed events. In particular, the meteors reported in Weryk \& Brown (2013b) primarily covered higher speeds; only half a dozen simultaneous radar-optical meteors had speeds below $20 \mathrm{~km} / \mathrm{s}$ among the analysed sample of 129 events, which had an average speed of $44 \mathrm{~km} / \mathrm{s}$. Moreover, most of these low speed events were well past the transition regime between underdense and overdense where the uncertainty in electron line density is higher. The resulting luminous efficiency and $\frac{\beta}{\tau}$ in Weryk \& Brown (2013b) are appropriate to speeds in excess of $20 \mathrm{~km} / \mathrm{s}$.

Our goal here is to estimate $\frac{\beta}{\tau}$ in the speed interval $10<\mathrm{v}<20 \mathrm{~km} / \mathrm{s}$ for the popu- 
lation of echoes which are in the underdense regime and hence fainter than the optical meteors reported by Weryk \& Brown (2013b). This is the range where we expect the most accurate estimates of electron line density to be possible. We make use of the recent improved laboratory measurements for $\beta$ reported by DeLuca et al. (2018) to provide a means to directly estimate luminous efficiency and hence meteoroid mass at low speeds.

\section{Data Collection}

\subsection{Overview}

Our simultaneous optical-radar meteor observations were made between mid-2017 and mid-2019, and the data reduction process and methodology is similar to our previous work (Weryk \& Brown, 2012, 2013b). Here we provide an overview of the key procedures used for analysis, highlighting the few differences from the earlier works, particularly with respect to optical measurements which were made with a new set of cameras.

The radar meteor data was collected by the Canadian Meteor Orbit Radar (CMOR) (Jones et al. 2005, Brown et al. 2008), while optical measurements were made using two new pairs of EMCCD cameras installed as a new instrument suite as part of the Canadian Automated Meteor Observatory (CAMO) (Weryk et al., 2013). CAMO consists of two identical automated optical stations separated by $45 \mathrm{~km}$, allowing optical triangulation of commonly observed meteor events. One of the CAMO stations is also co-located with the CMOR site; hence direct, common radar-optical measurements are possible.

CAMO runs automatically when clear, dark conditions are present. CAMO originally consisted of two distinct image intensified instrument suites. One was a mirror tracking system consisting of a wide-field intensified finder camera and a cued narrow field intensified camera attached to a telescope which viewed a pair of mirrors that tracked each meteor in real time. This system is collectively termed the "guided" system. Its design purpose was high temporal $(10 \mathrm{~ms})$ and spatial $(3 \mathrm{~m})$ resolution studies of faint $(+5)$ meteors. In addition to the guided system, is a wide-field fixed camera 
designed for population and flux studies to fainter meteor magnitudes $(+6.5)$, termed the influx system.

In 2016, four EMCCD cameras (two at each site) were installed at CAMO. This third optical system is optimized for population studies of faint, slow meteors, extending the sensitivity range of the influx system. As this is a new system for CAMO, we provide a detailed summary of the hardware and detection characteristics in section 2.3

EMCCD camera data were collected at each site and analysed to isolate individual meteor events. Commonly observed events are correlated after each nightly run, and trajectory solutions computed. Any of these optical meteor trajectories which were within $\pm 3^{\circ}$ of the specular point as seen from CMOR were flagged and the raw radar data centered around the time of the event (with a buffer of $\pm 5 \mathrm{sec}$ ) extracted and saved. The raw radar returns were then manually examined to search for echoes which had times within two seconds of the optical time estimate and interferometric locations within 3 degrees of the optically estimated specular point. This provided an initial filter to identify potential common optical-radar echoes.

After this initial coarse filter, only two station optical solutions having average speeds below $20 \mathrm{~km} / \mathrm{s}$ were selected for more detailed analysis. An optical meteor and radar echo were then positively associated as a common event if the following conditions were met:

1. The interferometry direction was within $1.5^{\circ}$ of the optical trail as measured from the EMCCD cameras at the CMOR site.

2. Radar range of the echo and the range computed from two station optical solutions for the specular point agreed to $<0.5 \mathrm{~km}$.

3. Timing of the radar peak power point and the frame containing two station optical solution for specular point are within $0.1 \mathrm{sec}$.

4. Two station optical solution must have an angle between the two observation planes larger than 10 degrees.

Using all automatically detected and computed EMCCD two station events collected between Oct 10, 2018 and May 10, 2019 (when the camera pointings were fixed 
and had common lenses and hence sensitivities) a total of 1249 possible optical events were selected in the first coarse filter as having a portion of their path within $3^{\circ}$ of the specular point. Of these, 96 had average optical speeds $<20 \mathrm{~km} / \mathrm{s}$, but only 14 passed all remaining criteria given above. In addition to these 14, a further 15 events (collected between July 1, 2017 and Oct 9, 2018) were added which also passed the correlation criteria above. These earlier events were collected at a time when the EMCCD systems were still in an engineering testing mode with some lens changes and minor changes made to the pointing directions. While the collecting characteristics were not identical in this period, the long collection time significantly improved number statistics at low speeds and these events are added to our study data set here as they are clearly common with echoes detected by CMOR. Our analysis consists of these 29 low speed events (to which we also added 7 higher speed events to provide some context for the lower speed measurements) for a total of 36 radar-optical low speed meteors examined in detail for our study.

\subsection{Radar Measurements}

Our specular radar measurements were made with the $29.85 \mathrm{MHz}$ system of the Canadian Meteor Orbit Radar (CMOR). CMOR is a triple frequency radar operating at $17.45,29.85$ and $38.15 \mathrm{MHz}$ having interferometric capability at each frequency. The 29.85 MHz system also has five additional remote sites which make common observations and permit 4000-5000 meteoroid orbits per day to be measured. Here we use only the data from the $29.85 \mathrm{MHz}$ system, with focus on the amplitude, range, and interferometry solution from the main site. We found that most of the low speed common optical events did not have measureable radar orbits in the normal CMOR orbit pipeline, consistent with our observation that most of the common echoes were near the radar detection limit. Hence we restrict our radar analysis to the received echo power at the main site. An example of such a radar echo record for a simultaneously observed optical meteor is shown in Figure 1. Details of the hardware, software, calibration and analysis pipeline are given in Webster et al. (2004); Jones et al. (2005); Brown et al. (2008); Weryk \& Brown (2012, 2013b).

The basic configuration for the radar is listed in Table 1 . The direction to each 


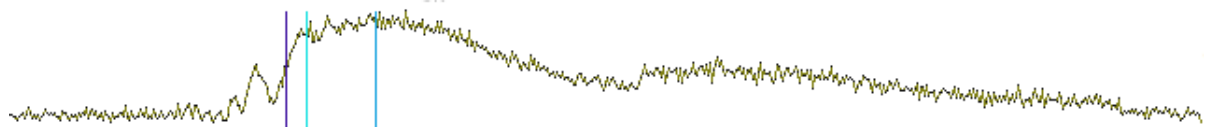

Figure 1: A raw radar range-time-intensity plot for the radar-optical meteor event detected on May 27, 2019 at 05:50:59 UTC. The top plot shows time on the abscissa - the entire axis as shown is $1.2 \mathrm{sec}$ in duration. The ordinate is range from the radar running from $15-255 \mathrm{~km}$. The brightness per pixel corresponds to the power received per sampled range gate. The echo at the main site is visible in the top plot - a horizontal slice of this echo power (in linear units) on the same time base is shown as the lower line plot. The vertical purple line is the estimated specular point while the light vertical blue line is the peak power point. The peak power point is the reference power level used to compute the electron line density representing the full power scattered from the trail after the meteor has fully traversed the first fresnel zone. This power is corrected for diffusion back to the specular point. The dark blue line shows the location of the absolute peak power. 
Table 1: Experimental configuration of CMOR for simultaneous radar-optical measurements. Here the total receiver and transmit gain in the direction of the fixed field of view EMCCD cameras is also shown, together with the equivalent limiting radar meteor magnitude in that direction.

\begin{tabular}{ll}
\hline \hline Quantity & Description \\
\hline Location & $43.264^{\circ} \mathrm{N}, 80.772^{\circ} \mathrm{W}, 324 \mathrm{~m}$ (WGS-84) \\
Frequency & $29.85 \mathrm{MHz}$ \\
Pulse duration & $75 \mu \mathrm{sec}$ \\
Pulse Repetition Frequency & $532 \mathrm{~Hz}$ \\
Range sampling interval & $15-255 \mathrm{~km}$ \\
Peak Transmitter power & $15 \mathrm{~kW}$ \\
Range accuracy & $<0.3 \mathrm{~km}$ \\
Total gain in FOV & $2.3-8.9 \mathrm{dBi}$ \\
Limiting equivalent radar meteor magnitude in FOV & $+5.7-+7.5$ \\
\hline
\end{tabular}

detected echo is found by interferometry using the phase differences (in pairs) between five antennas configured in a cross pattern as described in Jones et al. (1998). The interferometric accuracy is $\approx 0.8^{\circ}$ (Weryk \& Brown, 2012$)$. Timing is GPS conditioned and absolute times per recorded pulse are accurate to much better than the EMCCD interframe time of $30 \mathrm{~ms}$.

The main difference in radar data compared to the earlier work of Weryk \& Brown (2012, 2013b) is the higher transmit power (15 kW now versus $6 \mathrm{~kW}$ originally). As well, since raw radar returns are saved across all range gates for these echoes, the absolute range accuracy is higher than the range gate sampling interval $(3 \mathrm{~km})$ as the pulse shape is fit across each echo to isolate the peak range with an accuracy of $0.3 \mathrm{~km}$. Since the optical field of view is limited to a comparatively small fraction of the whole sky, the limiting sensitivity for EMCCD optical detections is below CMORs absolute detection limit as shown in Table 1 . 


\subsection{Optical data}

Optical measurements used four Nüvü HNü1024 EMCCD cameras 1 two at each CAMO site. The cameras are Peltier cooled to $-60^{\circ} \mathrm{C}$ and thermally regulated via liquid cooling. They are pointed in fixed directions each with a $15 \times 15$ degree field of view. Figure 2 shows a pair of cameras in situ. The cameras are GPS synchronized at the image stage and have absolute frame time accuracy better than $1 \mathrm{~ms}$. They have a midband spectral response, broadly comparable to the Gaia, G-band (Jordi et al. 2010), and we use G-band magnitudes throughout our analysis. The quantum efficiency of each camera is in excess of $90 \%$ between 500-700 nm. More details of the cameras are summarized in Table 2

Each CAMO site has two EMCCDs which are paired to observe common atmospheric volumes. The pair optimized for lower height events (cameras F) have a collecting area that is fairly flat with height (varying by less than a factor of two from 80-140 km altitude) versus the other pair (cameras G) which have a more pronounced atmospheric collecting area maximum centered near $105 \mathrm{~km}$. However, both pairs have significant collecting areas above $70 \mathrm{~km}$ with cameras $\mathrm{F}$ being slightly more sensitive than cameras $\mathrm{G}$ due to lower average ranges to events (higher pointing elevations). The limiting meteor detection sensitivity is near magnitude +8 , though portions of the lightcurve approaching magnitude +9 are measurable above the EMCCD background for some meteors. This is an order of magnitude smaller mass than than the +5 limiting optical sensitivity of Weryk \& Brown (2013b), and approaches or exceeds the radar sensitivity (which is about +7.5 equivalent magnitude) in the EMCCD field of view as seen from CMOR (Table 1).

The EMCCD image stream per camera is automatically searched using a modified cluster (blob) detector as a first stage for detection (Gural, 2016). A matched filter is applied in a second stage for automated refined astrometry and photometry estimations. Camera events are then correlated across sites using the EVCORR algorithm as part of the ASGARD detection package (Weryk et al. 2007) to produce trajectory and orbit solutions. Orbit counts per camera pair approach one per minute under good sky

1 http://www.nuvucameras.com/fr/files/2019/05/NUVUCAMERAS_HNu1024.pdf 
Table 2: Details of EMCCD. Pointing directions to the center of each field of view are given in degrees from the zenith $(\theta)$ and azimuth $(\varphi)$ measured $\mathrm{E}$ of $\mathrm{N}$.

\begin{tabular}{ll}
\hline & Specification \\
\hline CMOR Site [01] & $43.264^{\circ} \mathrm{N}, 80.772^{\circ} \mathrm{W}, 324 \mathrm{~m}(\mathrm{WGS}-84)$ \\
Elginfield Observatory [02] & $43.194^{\circ} \mathrm{N}, 81.316^{\circ} \mathrm{W}, 319 \mathrm{~m}$ ASL \\
Pointing 1F/1G $(\theta, \varphi)$ & $25^{\circ}, 323^{\circ} / 43^{\circ}, 330^{\circ}$ \\
Sensor & Teledyne e2v CCD201-20 \\
Pixels & $1024 \times 1024 @ 13 \mu \mathrm{m}$ \\
Digitization & $(14) / 16-\mathrm{bit}$ \\
Framerate $(1 \times 1 / 2 \times 2)$ & $16.7 / 32.7 \mathrm{fps}$ \\
Lens & $\mathrm{Nikkor} 50 \mathrm{~mm} \mathrm{f} / 1.2$ \\
Field-of-View & $14.7^{\circ} \times 14.7^{\circ}$ \\
Meteor Peak G-Magnitude Limit & 8.0 \\
Elginfield cameras F/G photometric offset & $-16.29 \pm 0.33 /-16.01 \pm 0.35$ \\
CMOR Site cameras F/G photometric offset & $-16.89 \pm 0.06 /-16.62 \pm 0.2$ \\
\hline
\end{tabular}

conditions. For this work, the automated solutions are used only as an initial filter for probable common optical-radar events. Once common events are identified and manually verified, all subsequent astrometric and photometric measurements are performed manually using the software METAL with its methods described by Weryk \& Brown (2013b).

\section{Methodology}

The core of our analysis leverages the fact that the ratio $\beta$ to $\tau$ can be estimated directly from simultaneous radar-optical meteor events. From the fundamental physical theory of meteor ablation, it is assumed that the instantaneous radiant power, I, is proportional to a fraction $\tau$ of the kinetic energy of mass-loss via (Ceplecha et al., 1998)

$$
I=\frac{\tau v^{2}}{2} \frac{d m}{d t}
$$




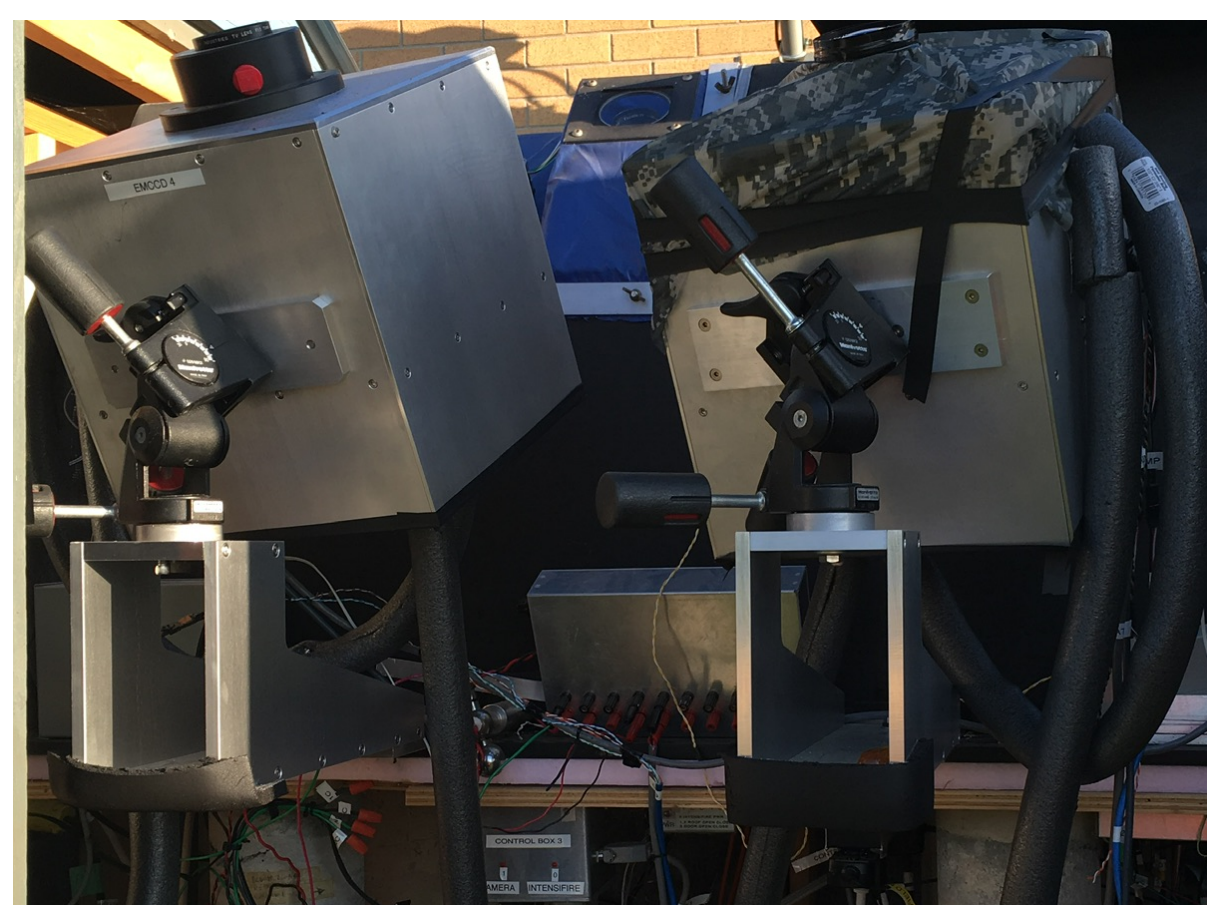

Figure 2: One pair of EMCCD cameras in-situ within the CAMO shed at the Elginfield site. 


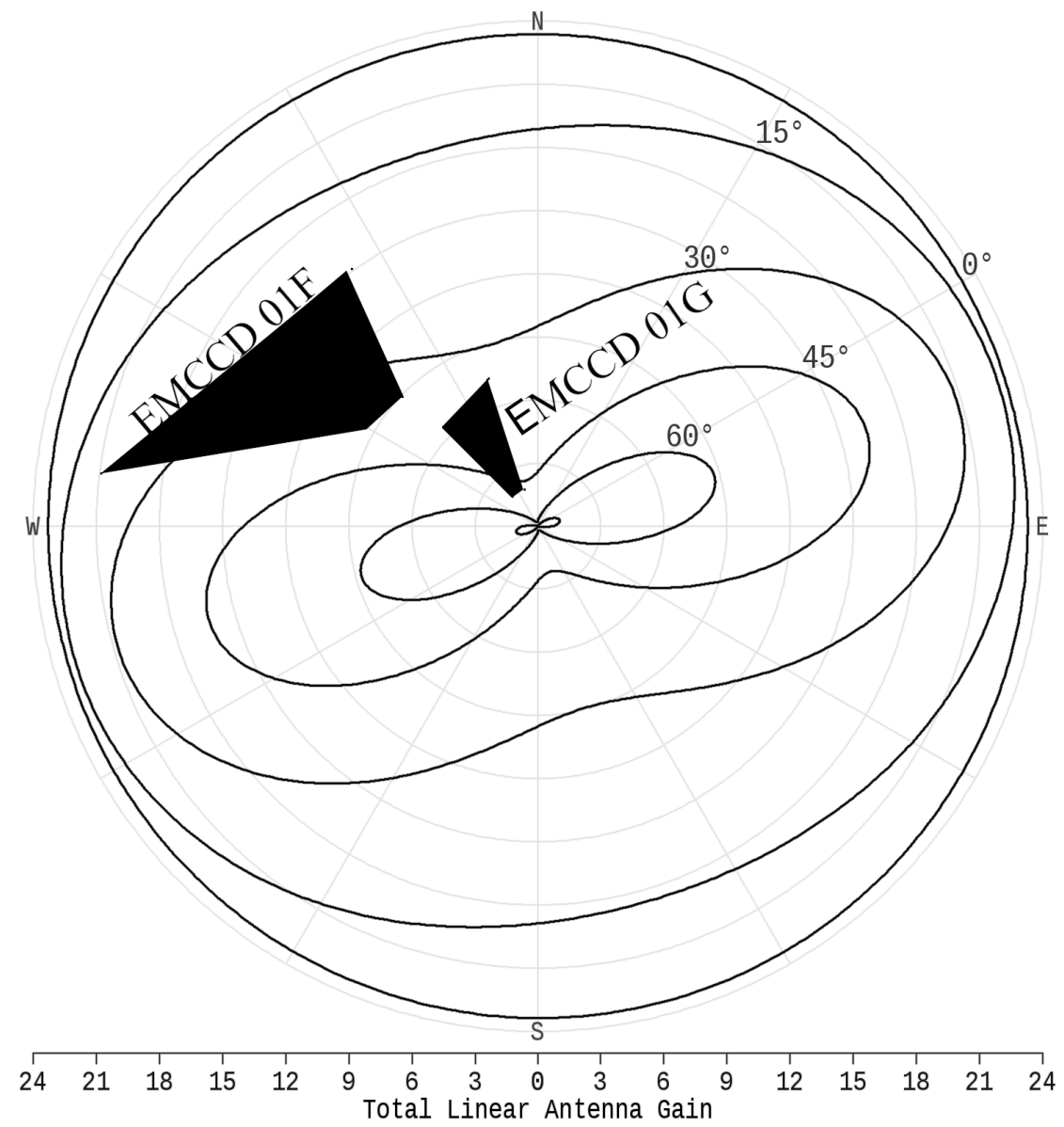

Figure 3: Field of view (FOV) of EMCCD cameras at the CMOR site projected on the sky with radar gain pattern overlay. The angular units show zenith distance as a function of azimuth with the horizon being at the center and the zenith around the edge of the circle. 


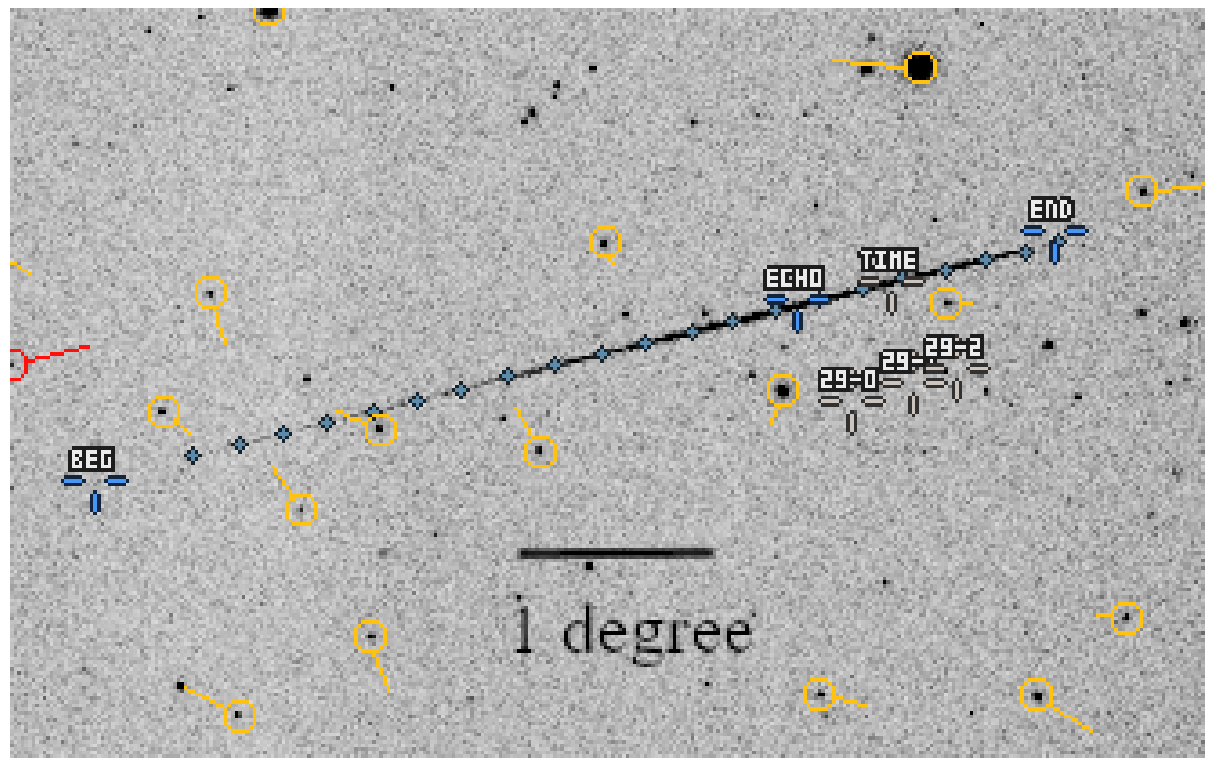

Figure 4: This meteor (20190527:055059) was detected by EMCCD 01F at the CMOR site and shows the full optical path from first detection (begin - in this case first seen from the other station) until final light (end). The individual blue symbols along the trajectory represent the manual astrometry picks per frame - this event lasted a total 20 EMCCD frames. The position marked "echo" is the two station optical solution estimate of the specular point - i.e. the location on the trail at $90^{\circ}$ from the apparent radiant as seen from the radar site. The time mark is the EMCCD time extracted from the radar when the echo specular point was reached. The "29-0" mark shows the estimated interferometric location of the echo as seen from the main site. The "29-1" and "29-2" marks represent the apparent location on the plane of the sky where the three station radar solution places the apparent path of the radar trajectory. Here an offset of $0.5^{\circ}$ between the optical and radar path is apparent, likely due to phase offsets at the main radar site. The timing and echo points are concordant within two video frames or $0.06 \mathrm{sec}$ while the 29-0 pick (the single station interferometric estimate of the echo location in the sky) is $0.5^{\circ}$ offset from the echo point, but parallel to the optical path. In this instance we have confidence from three independent measurements that the echo power is being produced along the lightcurve between the echo and time points, corresponding to an average absolute magnitude of +4.7 at the specular scattering point. 
where we have ignored the contribution due to deceleration.

Similarly, the number of free electrons produced per unit trail length, $q$, is commonly assumed to be proportional to a constant, $\beta$, times the mass loss rate (Ceplecha et al. 1998) giving

$$
q=\frac{\beta}{\mu v} \frac{d m}{d t}
$$

where $\mu$ is the average atomic mass of an ablated meteoroid atom, $v$ is the speed of the meteoroid, $q$ is the electron line density, and $I$ is the radiant power calibrated to our bandpass. We assume that $\mu$ is 24 amu consistent with a chondritic composition (Jarosewich, 1990).

Dividing Eq. 2 by 1 we can then relate the two constants, $\tau$ and $\beta$, through purely observed quantities as (Weryk \& Brown, 2013a)

$$
\frac{\beta}{\tau}=\frac{\mu v^{3} q}{2 I} .
$$

We compute the average velocity, $v$, from the two station optical solution using the Monte Carlo lag-weighting technique of Vida et al. (2019). The uncertainty in speed is taken to be the difference in speed between stations. The initial speed computed from this approach is also used to compute the meteoroid orbits (see Table 3). In most cases, even for our lowest speed events, the difference between the top of atmosphere initial speed and the average speed is found to be less than $1 \mathrm{~km} / \mathrm{s}$.

The radiant power per frame is computed by summing all the light from the meteor trail from the leading edge of the meteor back to the point of the leading edge from the previous frame after background subtraction. All photometry is calibrated to the Gaia $\mathrm{G}$ bandpass, with an assumed zero magnitude meteor having a radiant power of $945 \mathrm{~W}$ following Brown et al. (2017). This value is computed assuming meteor spectra can be fit as a $4500 \mathrm{~K}$ equivalent blackbody, an approximation found to be appropriate for slower meteors (Borovička, 1993, 1994, Ceplecha et al., 1998). For each meteor and each camera, a separate manual stellar photometric calibration was performed assuming the $\log$-sum-pixel intensity $\left(P_{i}\right)$ of a source is related to the $\mathrm{G}$ 
magnitude via:

$$
\mathrm{G}=\mathrm{C}-2.5 \log _{10} \sum \mathrm{P}_{\mathrm{i}}
$$

Here the zero point calibration $\mathrm{C}$ was found to be fairly constant over the two year period having a standard deviation among cameras for all events of between 0.06 and 0.35 magnitudes excluding the early intervals when engineering configurations were used, as shown in Table 2. A sample stellar photometric calibration is shown in Figure 5 . The meteor magnitude uncertainties per frame are the combined uncertainties from the stellar calibration zero point uncertainty and photon counting statistical uncertainty.

The final term to be measured in Eq. 3 is the electron line density, $q$. From the calibrated echo power received from the meteor as measured at the receivers $\left(P_{r}\right)$, we can relate the electron line density at our frequency via Kaiser \& Closs, 1952, Ceplecha et al., 1998):

$$
\mathrm{g}(\mathrm{q}, \mathrm{a})=\sqrt{\frac{P_{r} 32 \pi^{4} R^{3}}{P_{T} G_{T} G_{r} \lambda^{3}}}
$$

The terms on the right hand side are all known or directly measured and include $R$, the range to the meteor echo, $P_{T}$ the transmit power, $G_{T}$ and $G_{r}$ the antenna gain in the direction of the echo for the transmit and receive antennas respectively, and $\lambda$, the radar wavelength. From these quantities, the reflection coefficient, $g$, of the trail can be determined. Here $g$ represents the fraction of the incident electric field reflected by the trail, a value which can range from 0 to in excess of two (Poulter \& Baggaley, 1977) due to polarisation effects. The reflection coefficient is a function of both the electron line density and its radial distribution including the trail radius, a.

Estimating $q$ from $g$ requires a scattering model which depends on the orientation of the trail relative to the polarization direction of the radar pulse, a quantity which is known provided the trail direction is known (which is the case for all our optically measured events). It also depends on the radius, $a$, of the cylindrical trail column, the electron line density and the radial distribution of electrons in the trail. For the latter, a Gaussian distribution has traditionally been assumed (Kaiser \& Closs, 1952) a choice which is supported by more recent simulations (Jones, 1995). We estimate the initial radius as a function of height using the multi-frequency measurements from 


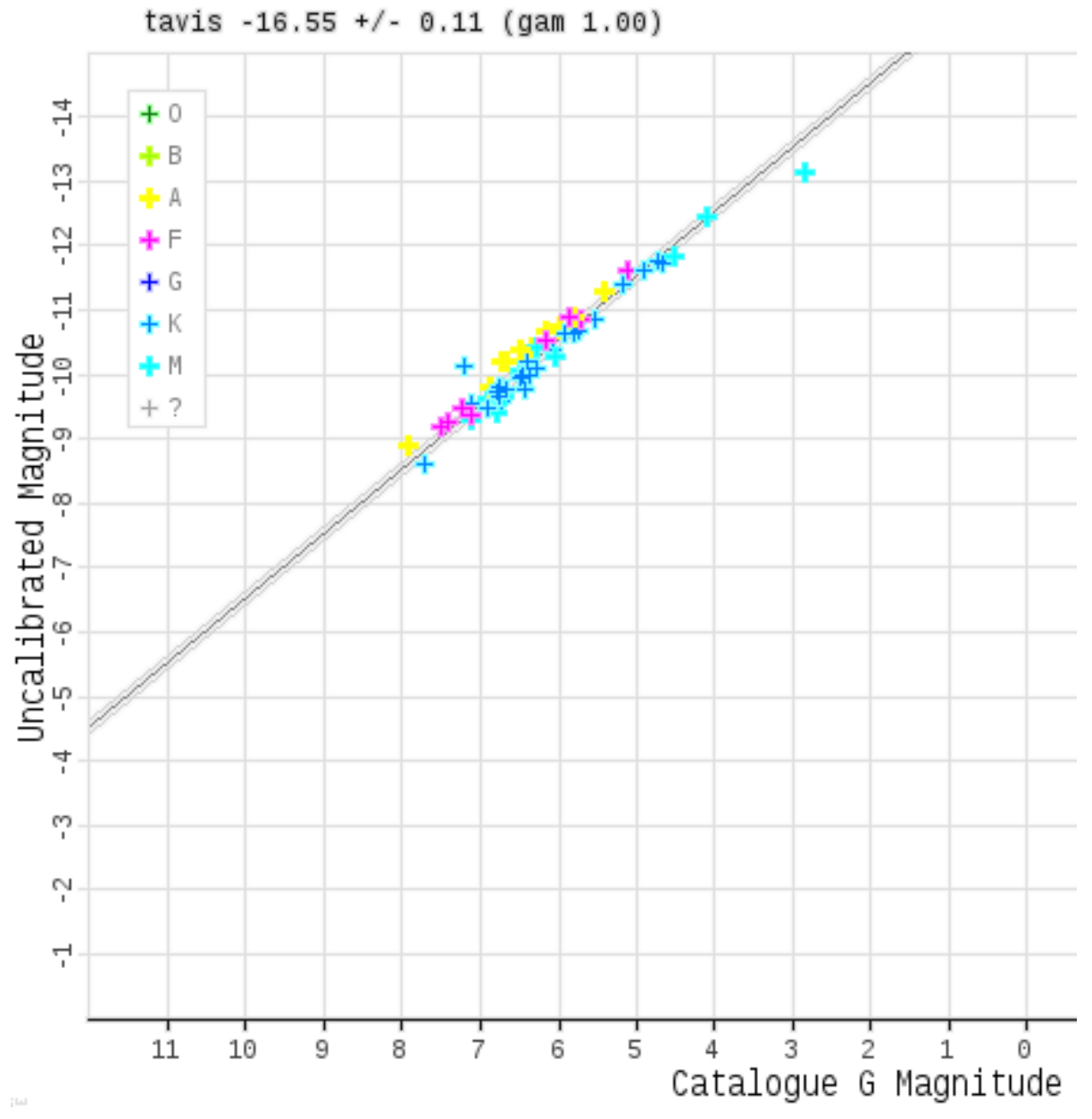

Figure 5: An example of a stellar photometric calibration for the CMOR EMCCD ' $G$ ' camera for the May 27, 055058 UTC event. The log sum pixel is shown on the ordinate and the calibrated G-band stellar magnitude is shown on the abscissa. For each star used in the calibration it's spectral type is shown as colored symbols which follow the legend inset. The zero point calibration value found through linear regression of the individual points (-16.55) plus its confidence bound (0.11) is also shown. 
CMOR reported by Jones \& Campbell-Brown (2005). We then compute the value of $q$ needed to produce the observed $g$ given the observed specular echo height (and hence trail radius corrected for ambi-polar diffusion) using the full wave scattering model of Poulter \& Baggaley (1977) as implemented through look-up tables computed by Weryk $\&$ Brown (2013b) for CMOR's wavelength.

\section{Results and Discussion}

Table 3 summarizes the measured velocities, orbits, and magnitudes at the specular point and corresponding electron line density for all our simultaneous radar-optical meteors. Also shown are the meteor begin and end heights from optical data as well as the height of the radar echo based on the interferometry and range. Note in three cases the radar height is slightly $(<0.5 \mathrm{~km})$ below the end height. This reflects the order of the uncertainty in the radar height; in these cases the specular point is taken to be at the end of the optical trail. The lightcurves for all events are shown in Appendix A

Using the data from Table 3 we compute the ratio of $\beta / \tau$ per event and its uncertainty, which reflects only our uncertainty in $v$ and $I$ - this is shown in Table 4 Figure 6 shows the resulting values of $\beta / \tau$ as a function of speed.

Our measured sample shows scatter in $\beta / \tau$ for a particular speed, but much of this variance is correlated with radiant intensity. In particular, the faintest events all have the largest $\beta / \tau$ as shown in Figure 7 The most recent lab experiments show no evidence for a variation of $\beta$ with mass at low speeds (DeLuca et al., 2018). Our results suggest that $\tau$ varies with radiant intensity as shown in Figure 8. This is consistent with the trend found in Weryk \& Brown (2013b), although their data was for brighter and faster meteors. It is the opposite trend found by Subasinghe \& Campbell-Brown (2018) and Capek et al. (2019), though in both cases they indicate that the trend is weak.

Examination of Table 3 shows that all of our simultaneous radar-optical meteors have orbits with $\mathrm{T}_{j}>3$. This suggests that our sample is dominated by asteroidal meteoroids. Among our sample of 36, six events each have abrupt onset lightcurves with low $(<20 \mathrm{~km} / \mathrm{s})$ speed, and are highlighted in the tables in bold. Such sudden onset lightcurves have been suggested as a likely feature of iron meteoroids by Capek et al. 
(2019). In their work, they identified probable iron meteoroids using a combination of spectral information and begin height/trail length criteria. In particular, through modelling, Čapek \& Borovička (2017) suggested that pure iron meteoroids should also begin ablation at lower heights than regular stony meteoroids and should show shorter trail lengths on average.

Figures 9 and 10 show the distribution of luminous begin heights and trail length as a function of speed for our events. The six sudden onset lightcurve meteors are shown in red. It is clear that these events have systematically lower begin heights than the rest of our population and are among the shortest path lengths. The values for trail length and begin height are in the range adopted by Capek et al. (2019) as representative of iron meteoroids. Figure 11 shows $\beta / \tau$ for only the lowest speed population (below 20 $\mathrm{km} / \mathrm{s}$ ) with the individual sudden-onset lightcurve events circled. The circled events we interpret as probable iron meteoroids. For such events, the only difference in measured $\beta / \tau$ would be that the average mass of ablated atoms should be $56 \mathrm{amu}$ (appropriate to iron) as opposed to our adopted chondritic average of $24 \mathrm{amu}$.

Since $\beta$ is larger for pure iron vs. chondritic bodies for a given speed, in Figure 12 we show the equivalent $\tau$ for all events assuming a pure iron composition for the six circled events and (for those six events) using the $\beta$ relation from DeLuca et al. (2018). All other events are assumed chondritic.

Our values for luminous efficiency show significant scatter, similar to other recent studies (eg. Weryk \& Brown (2013b); Subasinghe \& Campbell-Brown (2018); Capek et al. (2019). We find values of $\tau$ ranging from $<0.1 \%$ to as high as $30 \%$. There is no clear speed dependence at our low range of speeds, but there is a slight trend of higher $\tau$ with radiant intensity (see Figure 8), a result also found in Weryk \& Brown (2013b), though our number statistics are small so the significance of this trend is questionable. For our slow population (under $20 \mathrm{~km} / \mathrm{s}$ ) the average $\tau$ is $1.5 \%$, but this is misleading as there is a single larger outlier near 30\% (which is also one of our iron candidates). It is worth noting that for this iron candidate, reprocessing the measurements assuming it to be of chondritic composition drops $\tau$ to under 7\%. Removing this outlier, produces an average $\tau$ near $0.6 \%$, with a median value of $0.4 \%$, more representative of the slow population. The standard deviation of this group is also $0.6 \%$. From Figure 
12 our six iron meteoroids show $\tau$ values straddling the range given by the lab-based measurements of iron particles reported by Becker \& Friichtenicht (1971) .

Our events show luminous efficiencies below the values in our prior work (Weryk \& Brown, 2013b), but we suggest that this may in part be due to the differences in radiant power covered by the two studies as well as the limited speed range in the current work. The earlier study had detection limits roughly one order of magnitude brighter (in radiant power) than the current survey and higher $\tau$ for larger radiant powers are consistent with the trends we find.

The variance in $\tau$ almost certainly reflects, in part, the variation in the underlying spectra of the meteors in our sample. Our assumption of a G-band zero magnitude bolometric power of $945 \mathrm{~W}$ scaled to each meteor ignores this spectral diversity. To probe this relationship in more detail, spectral information, such as that used in the study of Vojáček et al. (2019) is required and would be highly desirable for an extension of the present work. Another source of scatter in $\tau$ may be fragmentation, which can affect the initial radius of meteor trails and ultimately our estimates of the electron line density (and ultimately $\tau$ ). Currently these estimates use the average initial radius model of Jones \& Campbell-Brown (2005).

The accuracy of the electron line densities estimated from the full wave modelling could be improved by performing multi-frequency fits to common echoes. In particular, a subset of slow meteors are detected at all three CMOR frequencies. For such events, model fits varying electron line density, trail radius and the diffusion coefficient to the complete amplitude - time echo records on all three frequencies would provide more stringent constraints and more robust uncertainties. We plan to examine such multifrequency common optical events in a future extension of the present work.

\section{Conclusions}

From a two year study capturing more than one thousand simultaneous opticalradar meteors, 36 events, mostly of low speed, were examined in detail. These meteors had G-band magnitudes of around +6 at the specular point and followed asteroidal orbits. These are the first in-situ radar-optical simultaneous measurements at such faint 


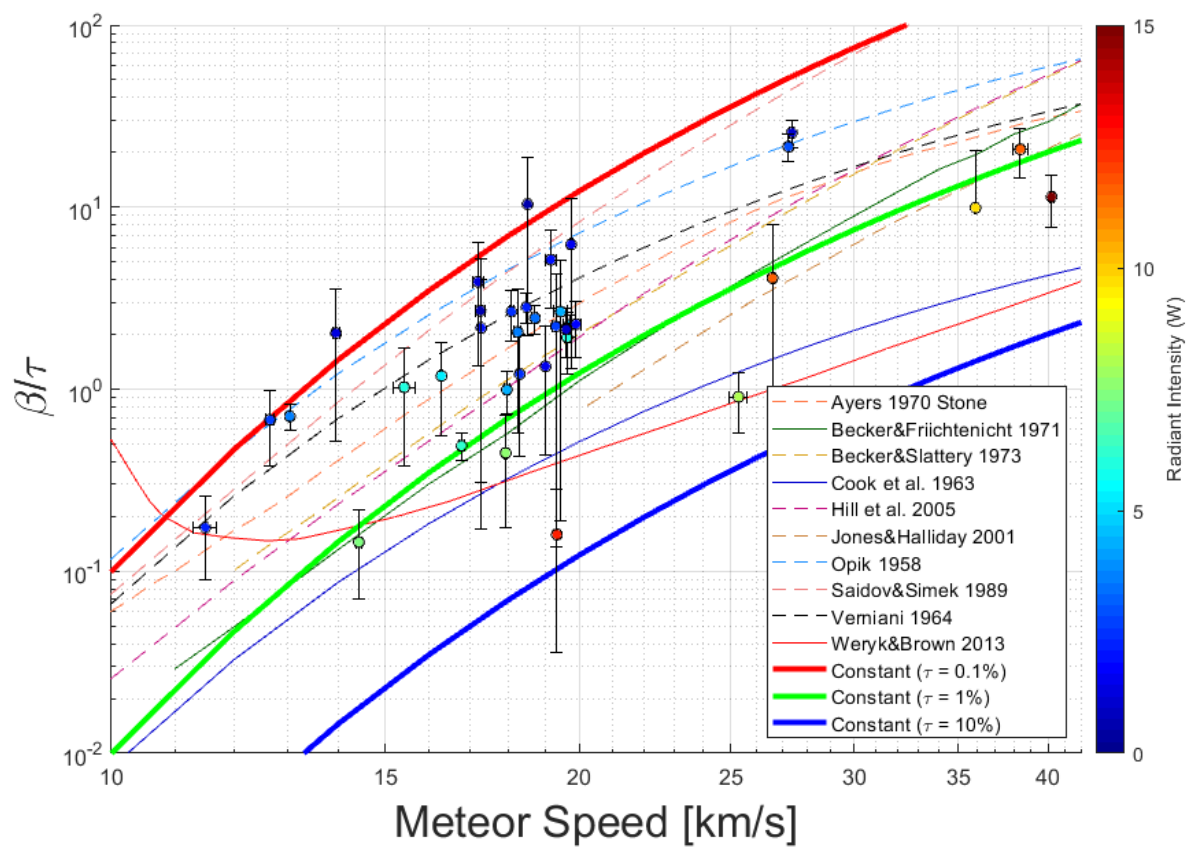

Figure 6: Measured values for $\beta / \tau$ for all 36 simultaneous radar-optical events as a function of speed. Each data point is color coded according to the radiant intensity at the specular point, where $10 \mathrm{~W}$ corresponds approximately to a +5 G-band magnitude meteor. Also shown for comparison are estimates of the ratio of $\beta / \tau$ derived from literature reporting various values for $\tau$. Here we use $\beta$ appropriate to a chondritic composition derived from Jones (1997) as parameterized in Weryk \& Brown (2013b) (their equation 15). Shown are estimates for $\tau$ for stony composition from Ayers et al. (1970), from laboratory measurements by Becker \& Friichtenicht (1971); Becker \& Slattery (1973), from small camera measurements of deceleration and luminosity from Cook et al. (1973); Verniani \& Hawkins (1964) and from theory by Opik (1955); Jones $\&$ Halliday (2001). Also shown are the hybrid theory-lab measurements estimates of $\tau$ from Hill et al. (2005) and direct estimates of the functional velocity form of $\beta / \tau$ from optical-radar measurements by Saidov $\&$ Simek (1989); Weryk \& Brown (2013b). Curves of constant $\tau$ are shown for comparison. 


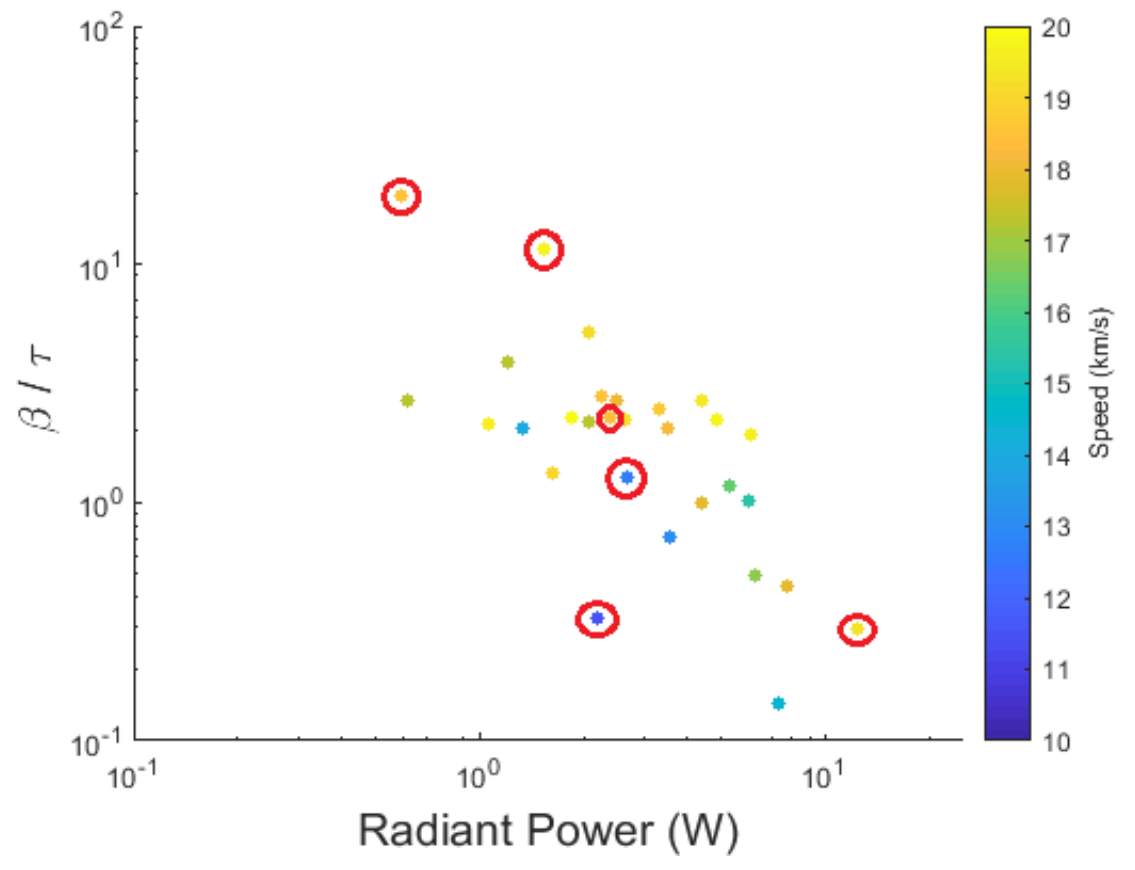

Figure 7: $\beta / \tau$ as a function of radiant intensity color coded by speed for low velocity $(<20 \mathrm{~km} / \mathrm{s})$ events only. Iron candidates are circled in red. 


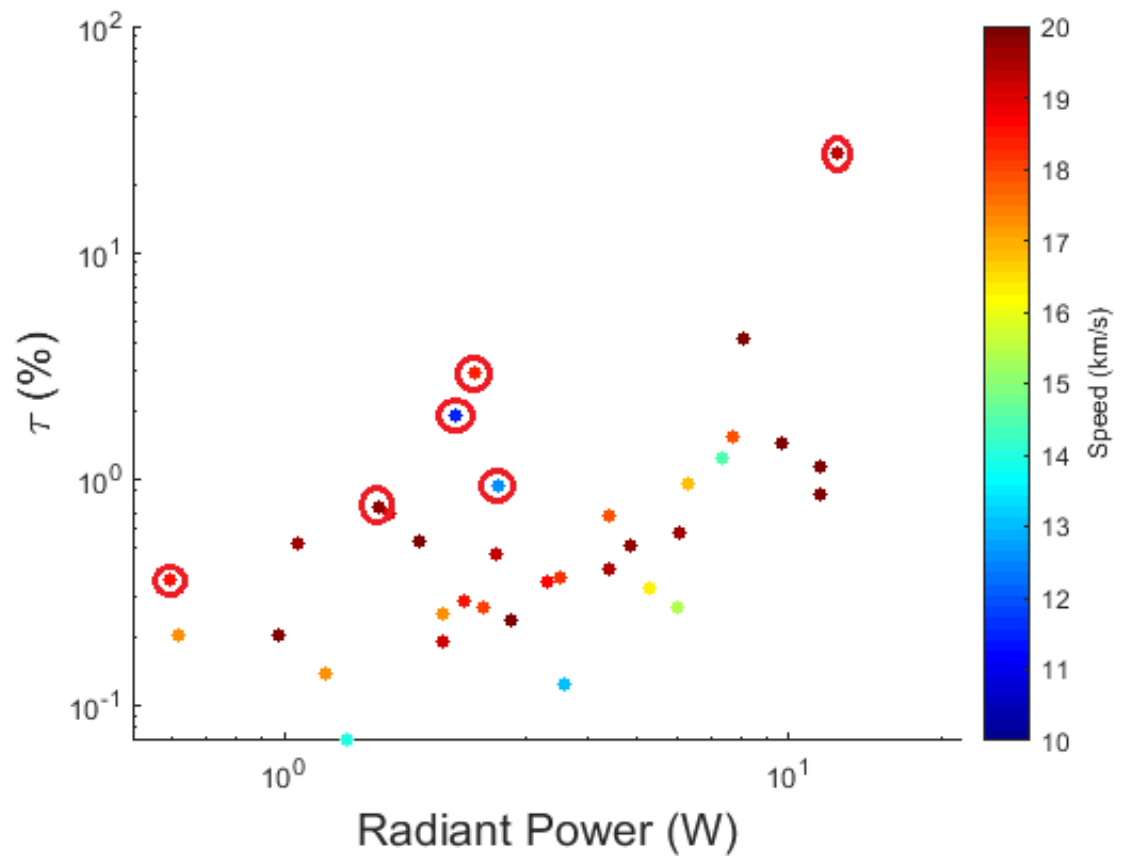

Figure 8: $\tau$ as a function of radiant intensity colour coded by speed for slow $(<20 \mathrm{~km} / \mathrm{s})$ events only. Here $\beta$ is assumed to be the chondritic mean value reported in Weryk \& Brown (2013b), except for our six probable iron meteoroids which use the iron $\beta$ from DeLuca et al. (2018). Iron candidates are circled in red. 


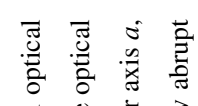

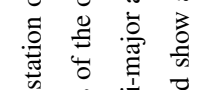

咅

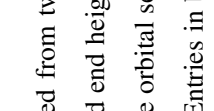

告

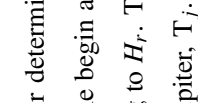

¿

峁 总

ङ

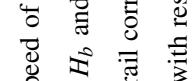

$\begin{array}{lll}0 & & \\ 0 & \vec{x} & 0 \\ 0 & 0 & \end{array}$

苛艺

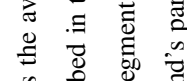

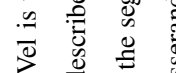

s

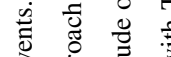

ठ 岕 营

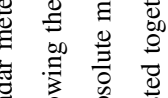

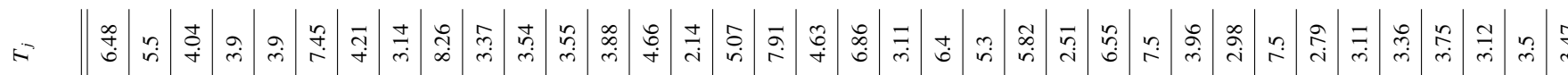

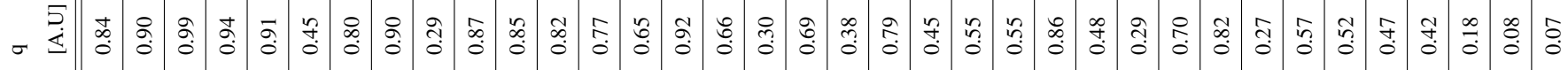

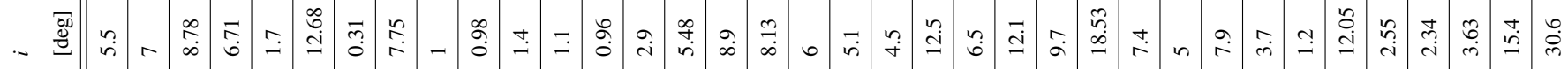

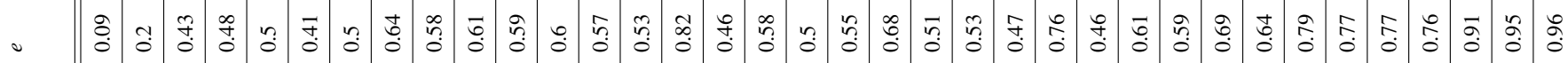

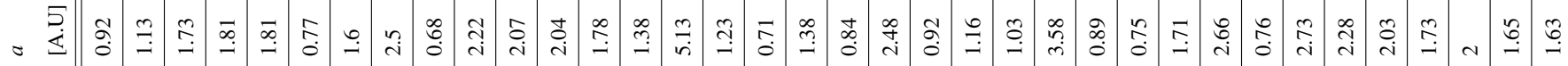

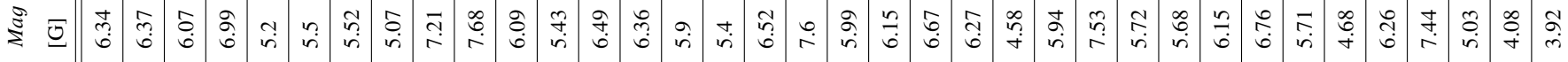

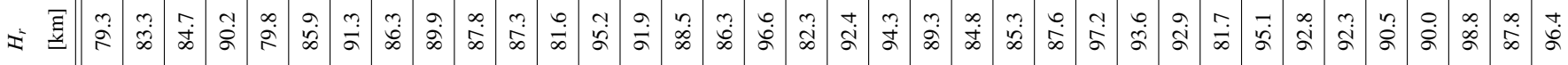

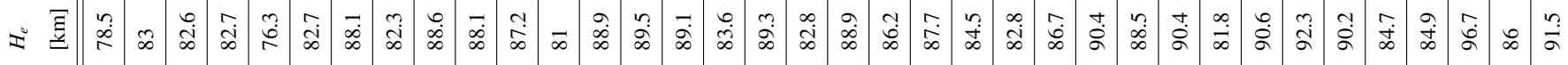

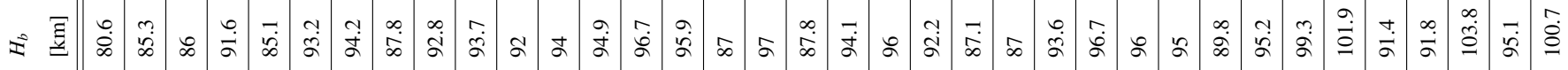

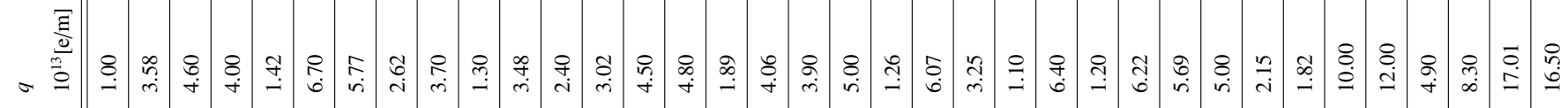

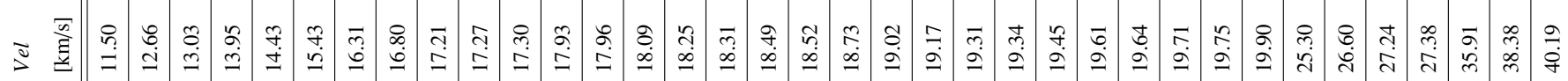

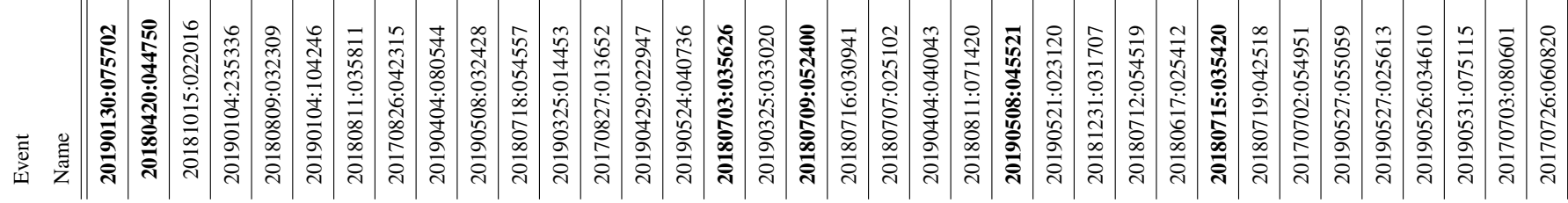




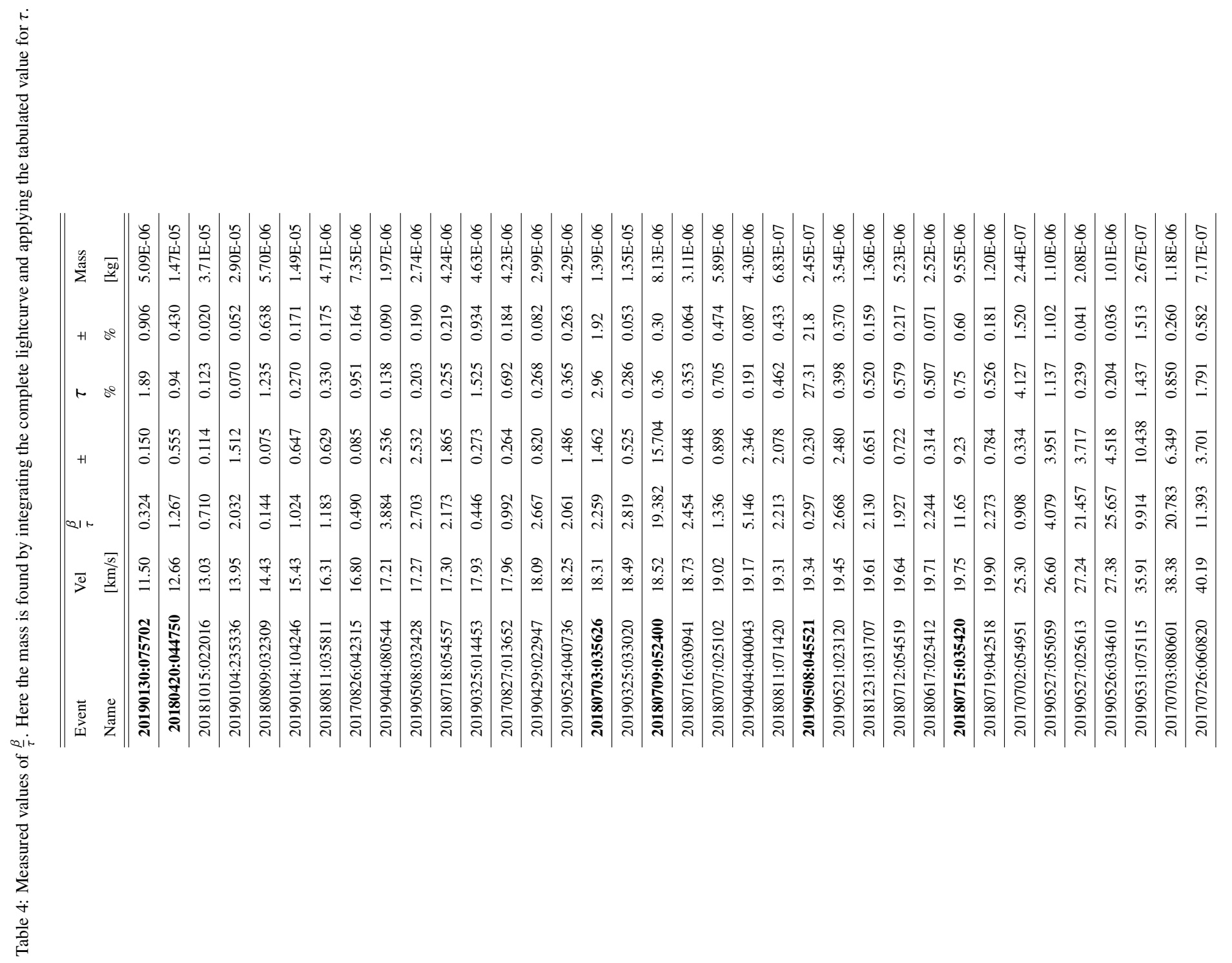




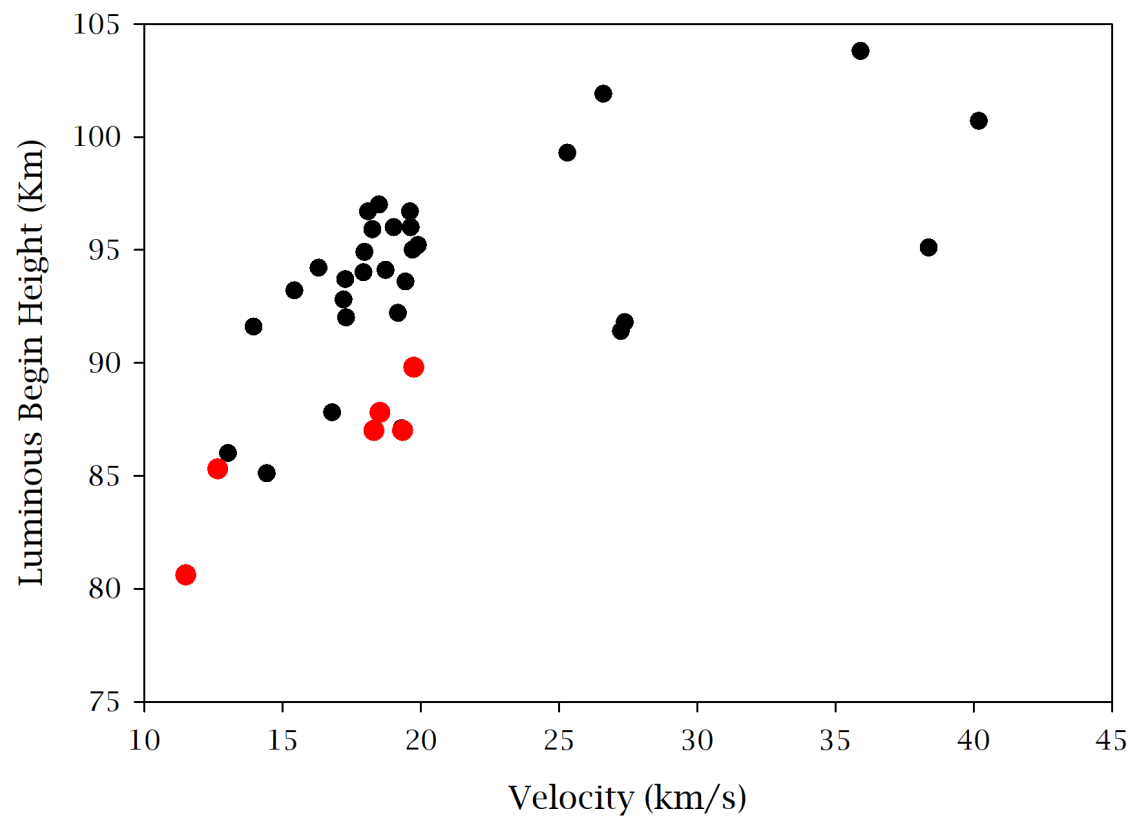

Figure 9: Begin height as a function of initial speed for all events in our dataset. As we required complete lightcurves from all events these represent the true initial heights - ie. the height at which luminosity first exceeds the instrument limit. Red symbols represent the six events which display sudden onset lightcurves. 


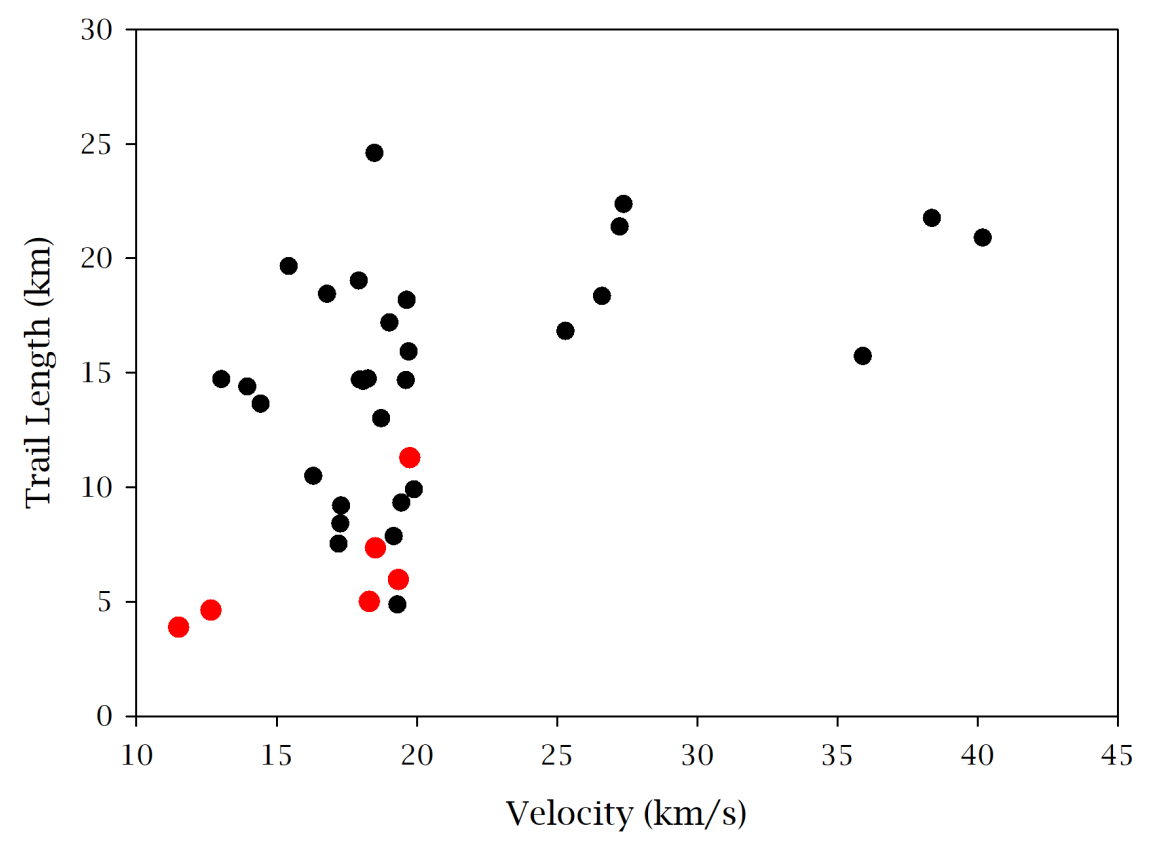

Figure 10: Total meteor trail length as a function of speed. This represents the total length of luminous flight above the detection limit of the instrument (near magnitude +9 ). Red symbols represent the six events which display sudden onset lightcurves. 


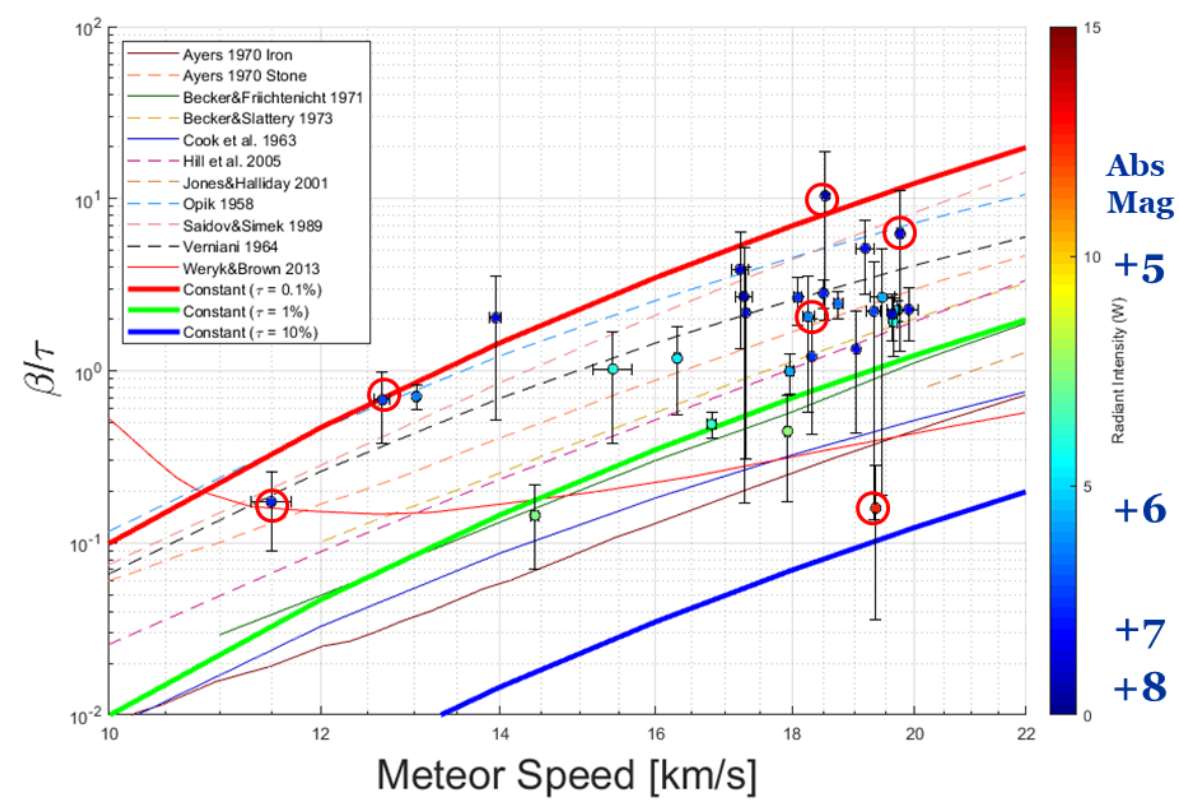

Figure 11: Measured values for $\beta / \tau$ for all 28 simultaneous radar-optical events with velocities below 20 $\mathrm{km} / \mathrm{s}$ as a function of speed. Here $\beta$ is appropriate to a chondritic composition. Each data point is colour coded according to the radiant intensity at the specular point. The reference curves are the same as in Figure 6 Circled points (in red) are meteors which have abrupt onset lightcurves, potentially consistent with an iron composition. 


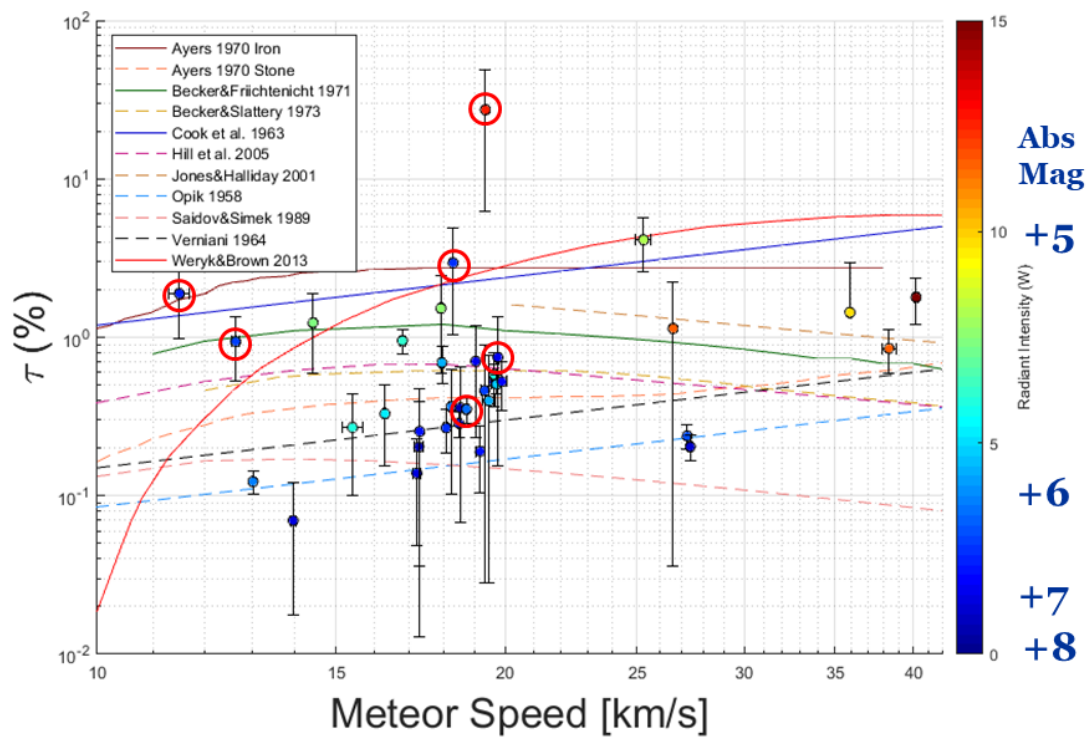

Figure 12: Best estimate for $\tau$ for all events. For circled events, values for $\beta$ appropriate to iron (from DeLuca et al. (2018)) are used. Each meteor is colour-coded by the radiant intensity at the specular point. 
magnitudes and low speeds and therefore represent a critical check on laboratory measurements of both $\beta$ (DeLuca et al., 2018) and $\tau$ (Tarnecki et al., 2019).

Our major conclusions are:

1. The ratio of $\beta / \tau$ shows a strong negative correlation with radiant power. No correlation is found with speed.

2. Among our slow $(<20 \mathrm{~km} / \mathrm{s})$ population, some $20 \%$ showed characteristics consistent with pure iron meteoroids.

3. The G-band luminous efficiencies at low speeds show considerable scatter, but average $0.6 \% \pm 0.6 \%$, with a median value of $0.4 \%$.

4. A slight positive correlation of $\tau$ with speed was identified.

\section{Acknowledgements}

This work was supported in part by the NASA Meteoroid Environment Office under cooperative agreement 80NSSC18M0046. PGB also acknowledges funding support from the Natural Sciences and Engineering Research council of Canada and the Canada Research Chairs program. We thank Z. Krzeminski for help in optical data reduction and J. Gill, M. Mazur for software support and camera operations.

\section{References}

Ayers, W., McCrosky, R., \& Shao, C. C.-Y. (1970). Photographic observations of 10 artificial meteors. SAO Spec. Rep \#317, 317, 40.

Becker, D. G., \& Friichtenicht, J. F. (1971). Measurement and interpretation of the luminous efficiencies of iron and copper simulated micrometeors. Astrophysical Journal, 166.

Becker, D. G., \& Slattery, J. (1973). Luminous efficiency measurements for silicon and aluminum simulated micrometeors. Astrophysical Journal, 186, 1127-1139.

Borovička, J. (1993). A fireball spectrum analysis. Astronomy and Astrophysics, 279, $627-645$. 
Borovička, J. (1994). Two components in meteor spectra. Planetary and Space Science, $42,145-150$.

Borovička, J., Koten, P., Spurný, P., Bocek, J., \& Stork, R. (2005). A survey of meteor spectra and orbits: evidence for three populations of Na-free meteoroids. Icarus, $174,15-30$.

Brown, P. G., Stober, G., Schult, C., Krzeminski, Z., Cooke, W., \& Chau, J. (2017). Simultaneous optical and meteor head echo measurements using the Middle Atmosphere Alomar Radar System (MAARSY): Data collection and preliminary analysis. Planetary and Space Science, 141, 25-34.

Brown, P. G., Weryk, R., Wong, D., \& Jones, J. (2008). A meteoroid stream survey using the Canadian Meteor Orbit Radar I. Methodology and radiant catalogue. Icarus, 195, 317-339.

Campbell-Brown, M. D. (2015). A population of small refractory meteoroids in asteroidal orbits. Planetary and Space Science, 118, 8-13.

Campbell-Brown, M. D., Kero, J., Szasz, C., Pellinen-Wannberg, a., \& Weryk, R. (2012). Photometric and ionization masses of meteors with simultaneous EISCAT UHF radar and intensified video observations. Journal of Geophysical Research, $117,1-13$.

Čapek, D., \& Borovička, J. (2017). Ablation of small iron meteoroids-First results. Planetary and Space Science, 143, 159-163.

Capek, D., Koten, P., Borovička, J., Vojáček, V., Spurný, P., \& Štork, R. (2019). Small iron meteoroids. Astronomy $\mathcal{E}$ Astrophysics, 625, A106.

Carrillo-Sánchez, J. D., Nesvorný, D., Pokorný, P., Janches, D., \& Plane, J. (2016). Sources of cosmic dust in the Earth's atmosphere. Geophysical Research Letters, 43, 979-11.

Ceplecha, Z., Spalding, R. E., Jacobs, C., ReVelle, D., Tagliaferri, E., \& Brown, P. G. (1998). Superbolides. Meteoroids 1998, (p. 37-54). 
Close, S., Oppenheim, M. M., Hunt, S., \& Coster, A. (2004). A technique for calculating meteor plasma density and meteoroid mass from radar head echo scattering. Icarus, $168,43-52$.

Cook, A., FORTI, G., McCrosky, R., Posen, A., Southworth, R. B., \& WILLIAMS, J. (1973). Combined observations of meteors by image-orthicon television camera and multi-station radar(to compare ionization with luminosity). In NASA, Washington Evolutionary and Phys. Properties of Meteoroids p 23-44(SEE N 74-19436 10-30).

DeLuca, M., Munsat, T., Thomas, E., \& Sternovsky, Z. (2018). The ionization efficiency of aluminum and iron at meteoric velocities. Planetary and Space Science, $156,111-116$.

Friichtenicht, J. F., Slattery, J., \& E (1968). A laboratory measurement of meteor luminous efficiency. The Astrophysical, 151, 747-758.

Gural, P. (2016). A fast meteor detection algorithm. In International Meteor Conference Egmond, the Netherlands, 2-5 June 2016 (pp. 96-104).

Hill, K., Rogers, L., \& Hawkes, R. (2005). High geocentric velocity meteor ablation. Astronomy and Astrophysics, 444, 615-624.

Jarosewich, E. (1990). Jarosewich (1990) - Chemical analyses of meteorites- A compilation of stony and iron meteorite analyses. Meteoritics, 25, 323-337.

Jones, J., Brown, P. G., Ellis, K. J., Webster, A., Campbell-Brown, M. D., Krzemenski, Z., \& Weryk, R. (2005). The Canadian Meteor Orbit Radar : system overview and preliminary results. Planetary and Space Science, 53, 413-421.

Jones, J., \& Campbell-Brown, M. D. (2005). The initial train radius of sporadic meteors. Mon. Not. R. Astron. Soc, 359, 1131-1136.

Jones, J., Webster, A., \& Hocking, W. (1998). An improved interferometer design for use with meteor radars. Radio Science, 33, 55-65.

Jones, W. (1995). Theory of the initial radius of meteor trains. Monthly Notices of the Royal Astronomical Society, 275, 812-818. 
Jones, W. (1997). Theoretical and observational determinations of the ionization coefficient of meteors. Monthly Notices of the Royal Astronomical Society, 288, 995-1003.

Jones, W., \& Halliday, I. (2001). Effects of excitation and ionization in meteor trains. Monthly Notices of the Royal Astronomical Society, 320, 417-423.

Jordi, C., Gebran, M., Carrasco, J. M., de Bruijne, J., Voss, H., Fabricius, C., Knude, J., Vallenari, A., Kohley, R., \& Mora, A. (2010). Gaia broad band photometry. Astronomy $\mathcal{E}$ Astrophysics, 523, A48.

Kaiser, T., \& Closs, R. L. (1952). Theory of radio reflections from meteor trails: I. Phil Mag, 43, 1-32.

Klekociuk, A. R., Brown, P. G., Pack, D., ReVelle, D., Edwards, W., Spalding, R. E., Tagliaferri, E., Yoo, B. B., \& Zagari, J. (2005). Meteoritic dust from the atmospheric disintegration of a large meteoroid. Nature, 436, 1132-5.

Liou, J., Dermott, S., \& Xu, Y. (1995). The contribution of cometary dust to the zodiacal cloud. Planetary and Space Science, 43, 717-722.

Marshall, R. A., Brown, P. G., \& Close, S. (2017). Plasma distributions in meteor head echoes and implications for radar cross section interpretation. Planetary and Space Science, (pp. 1-6).

Mathews, J., Briczinski, S., Malhotra, A., \& Cross, J. (2010). Extensive meteoroid fragmentation in V/UHF radar meteor observations at Arecibo Observatory. Geophysical Research Letters, 37, 1-5.

Nesvorný, D., Jenniskens, P., Levison, H., Bottke, W., Vokrouhlický, D., \& Gounelle, M. (2010). Cometary Origin of the Zodiacal Cloud and Carbonaceous Micrometeorites. Implications for Hot Debris Disks. The Astrophysical Journal, 713, 816-836.

Nishimura, K., Sato, T., Nakamura, T., \& Ueda, M. (2001). High Sensitivity RadarOptical Observations of Faint Meteors. IEICE Trans Commun, E84-C, 1877-1884. 
Opik, E. J. (1955). Meteor radiation, ionization and atomic luminous efficiency. Proceedings of the Royal Society of London. Series A, Mathematical and Physical Sciences, 230, 463-501.

Poulter, E., \& Baggaley, W. (1977). Radiowave scattering from meteoric ionization. Journal of Atmospheric and Terrestrial Physics, 39, 757-768.

Saidov, K., \& Simek, M. (1989). Luminous efficiency coefficient from simultaneous meteor observations. Bulletin of the Astronomical Institutes of Czechoslovakia, 40, $330-332$.

Silber, E., Brown, P. G., \& Krzeminski, Z. (2015). Optical observations of meteors generating infrasound: Weak shock theory and validation. Journal of Geophysical Research: Planets, (pp. 413-428).

Slattery, J., \& Friichtenicht, J. F. (1967). Ionization probability of iron particles at meteoric velocities. The Astrophysical Journal, 147.

Subasinghe, D., \& Campbell-Brown, M. (2018). Luminous Efficiency Estimates of Meteors. II. Application to Canadian Automated Meteor Observatory Meteor Events. The Astronomical Journal, 155, 88.

Subasinghe, D., Campbell-Brown, M. D., \& Stokan, E. (2016). Physical characteristics of faint meteors by light curve and high-resolution observations, and the implications for parent bodies. Monthly Notices of the Royal Astronomical Society, 457, 12891298.

Subasinghe, D., Campbell-Brown, M. D., \& Stokan, E. (2017). Luminous efficiency estimates of meteors -I. Uncertainty analysis. Planetary and Space Science, 143, $71-77$.

Tarnecki, L. K., Marshall, R. A., Sternovsky, Z., Munsat, T. L., \& DeLuca, M. (2019). Laboratory Dust Ablation Experiments to Characterize Meteoric Luminous Efficiencies. In AGUFM (pp. P21F-3436). volume 2019. 
Thomas, E., Horányi, M., Janches, D., Munsat, T., Simolka, J., \& Sternovsky, Z. (2016). Measurements of the ionization coefficient of simulated iron micrometeoroids. Geophysical Research Letters, 43, 3645-3652.

Verniani, F., \& Hawkins, G. (1964). On the ionizaing efficiency of meteors. Astrophysical Journal, 140, 1590.

Vida, D., Gural, P. S., Brown, P. G., Campbell-Brown, M., \& Wiegert, P. (2019). Estimating trajectories of meteors: an observational Monte Carlo approach - I. Theory. Monthly Notices of the Royal Astronomical Society, 19, 1-19.

Vojáček, V., Borovička, J., Koten, P., Spurný, P., \& Štork, R. (2019). Properties of small meteoroids studied by meteor video observations. Astronomy and Astrophysics, 621, $1-21$.

Webster, A., Brown, P. G., Jones, J., Ellis, K. J., \& Campbell-Brown, M. D. (2004). Canadian Meteor Orbit Radar (CMOR). Atmospheric Chemistry and Physics, 4, 679-684.

Weryk, R., \& Brown, P. G. (2012). Simultaneous radar and video meteors-I: Metric comparisons. Planetary and Space Science, 62, 132-152.

Weryk, R., \& Brown, P. G. (2013a). Simultaneous radar and video meteors - II: Photometry and ionisation. Planetary and Space Science, 81, 32-47.

Weryk, R., Brown, P. G., Domokos, A., Edwards, W., Krzeminski, Z., Nudds, S. H., \& Welch, D. L. (2007). The Southern Ontario All-sky Meteor Camera Network. Earth, Moon, and Planets, 102, 241-246.

Weryk, R., Campbell-Brown, M. D., Wiegert, P., Brown, P. G., Krzeminski, Z., \& Musci, R. (2013). The Canadian Automated Meteor Observatory (CAMO): System overview. Icarus, 225, 614-622.

Weryk, R. J., \& Brown, P. G. (2013b). Simultaneous radar and video meteors-II: Photometry and ionisation. Planetary and Space Science, 81, 32-47. 
Yang, H., \& Ishiguro, M. (2015). Origin of Interplanetary Dust Through Optical Properties of Zodiacal Light. The Astrophysical Journal, 813, 87. 


\section{Appendix A. Lightcurves}

In the following pages are plots of the absolute brightness of each meteor as a function of the EMCCD camera time from both stations for common events. In some cases more than two cameras detected the same event; in those cases all three (or four) lightcurves are shown. Note that the radar site is located at Tavistock. The name convention for each event is the time of appearance as YYYYMMDD:HHMMSS in UTC. 


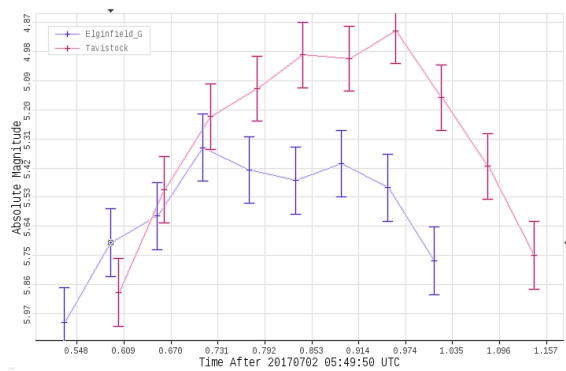

20170702:054951

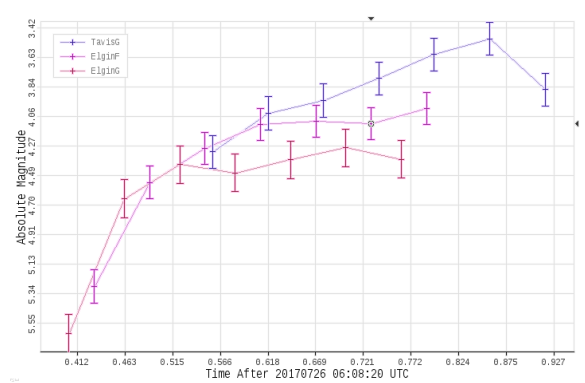

20170726:060820

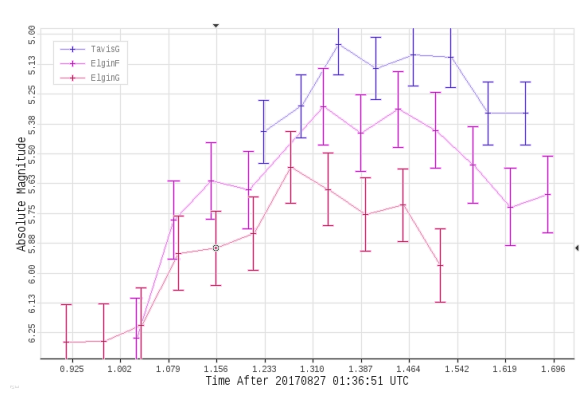

20170827:013651

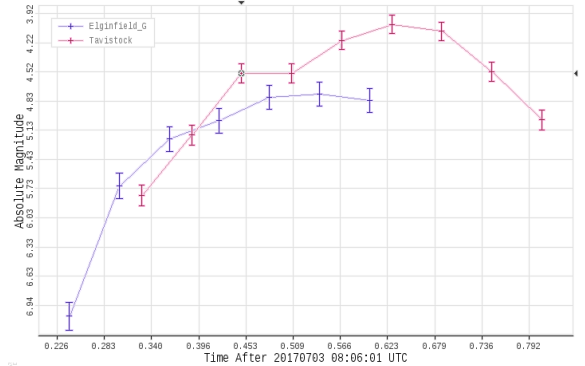

20170703:080601

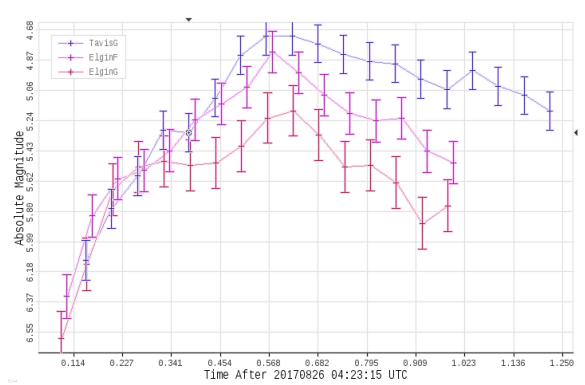

20170826:04231

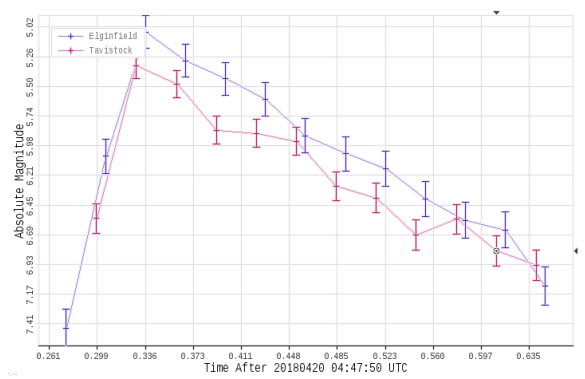

20180420:044750 


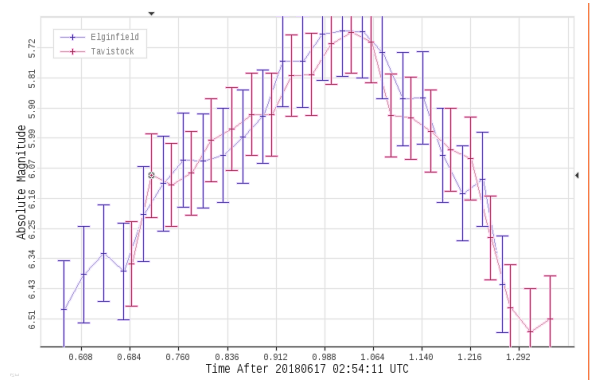

20180617:025411

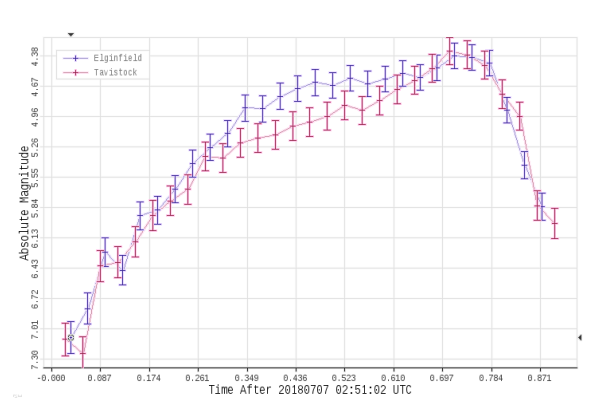

20180617:025411

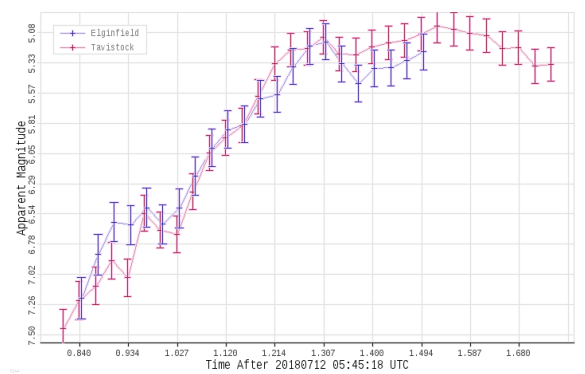

20180712:054518

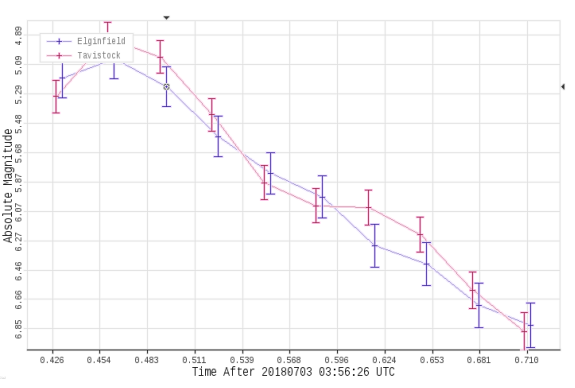

20180703:035626

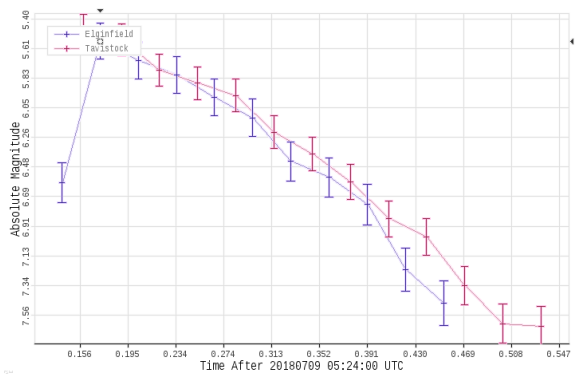

20180709:052400

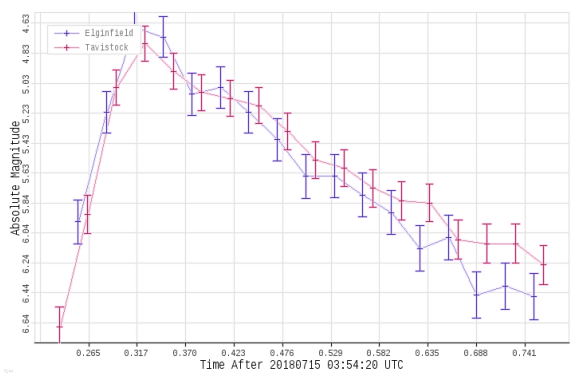

20180715:035420 


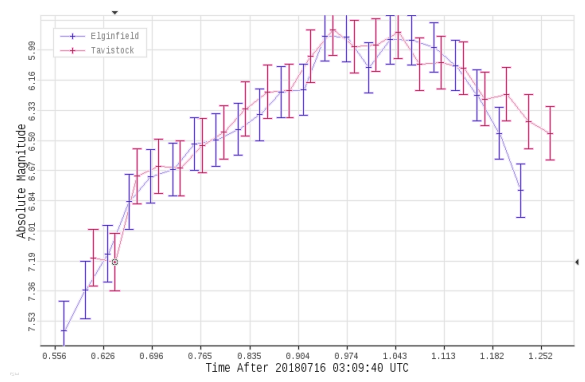

20180716:030940

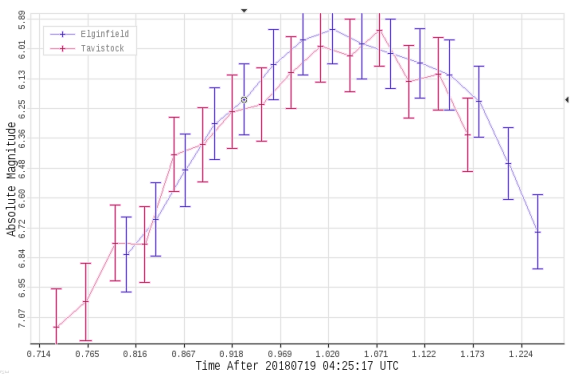

20180719:042517

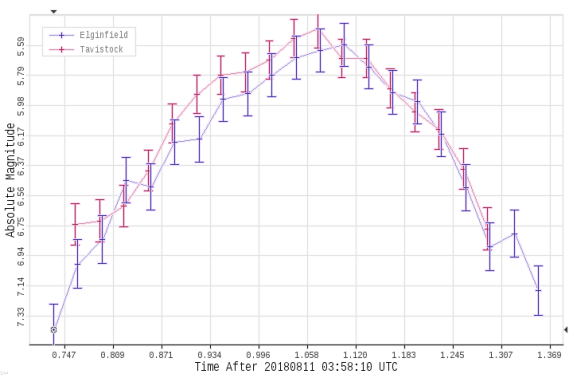

20180811:035810

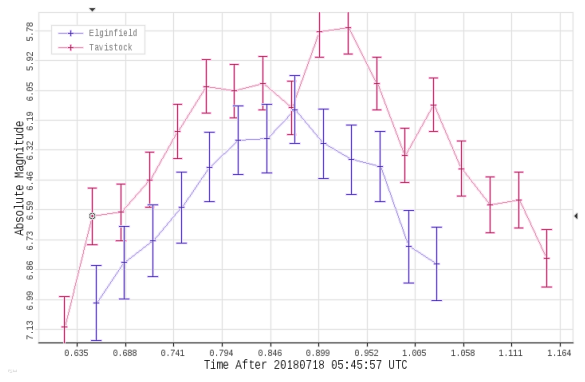

20180718:054557

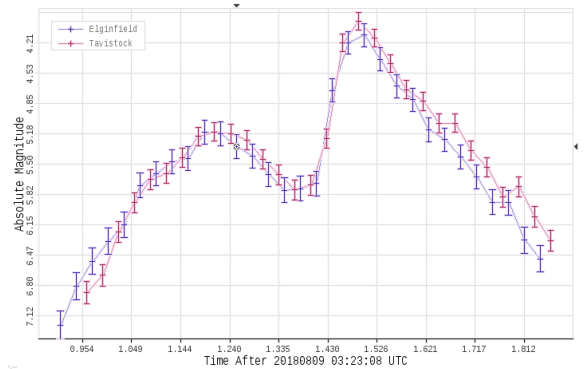

20180809:032308

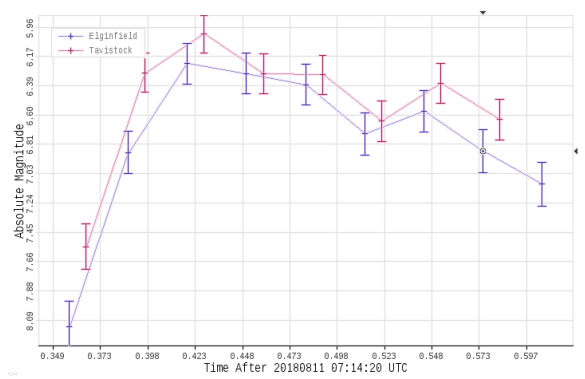

20180811:071420 


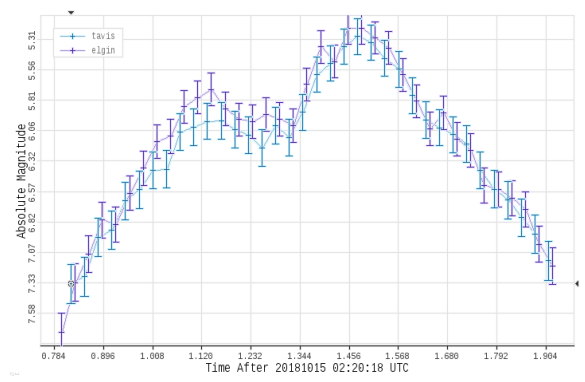

20181015:022018

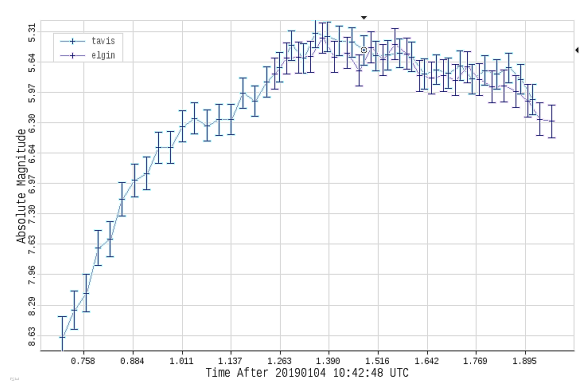

20190104:104248

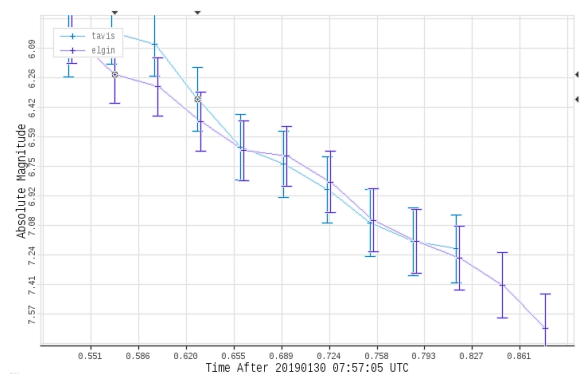

20190130:07570

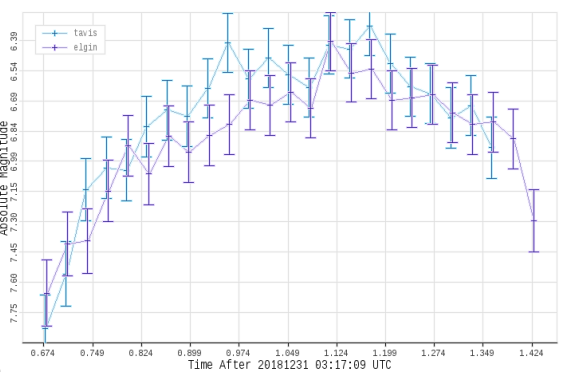

20181231:031709

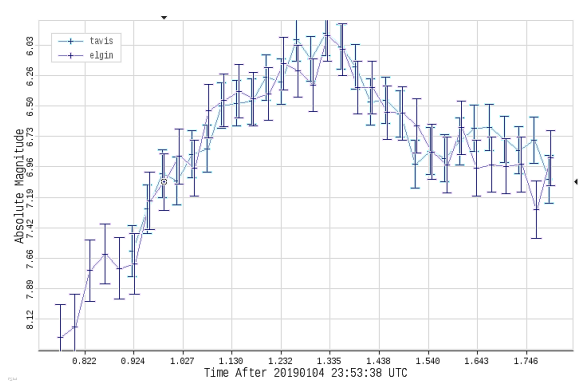

20190104:235338

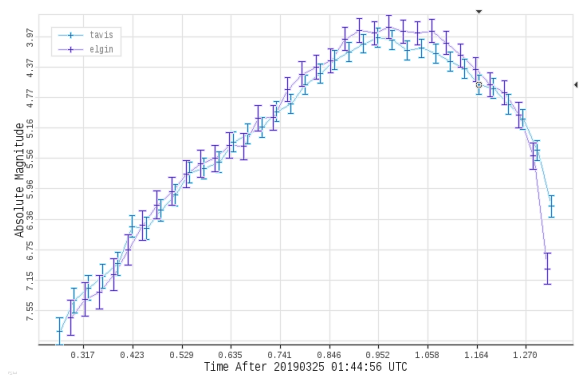

20190325:014456 

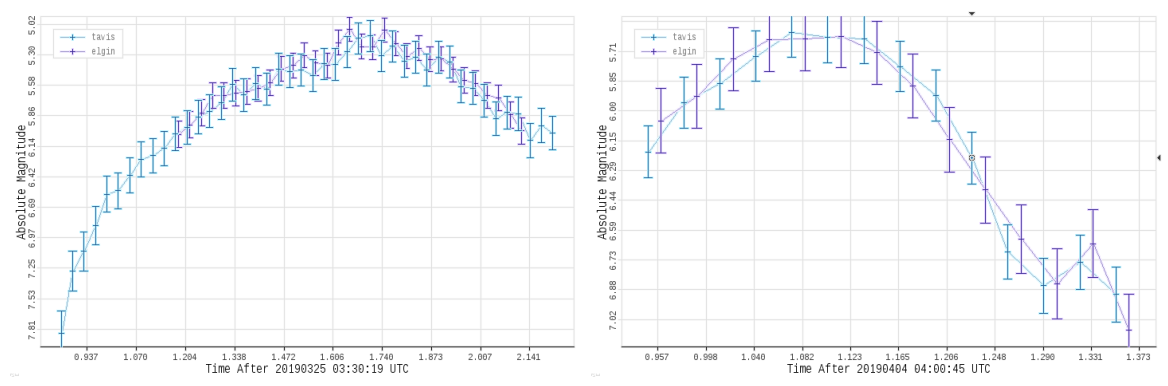

20190325:033019

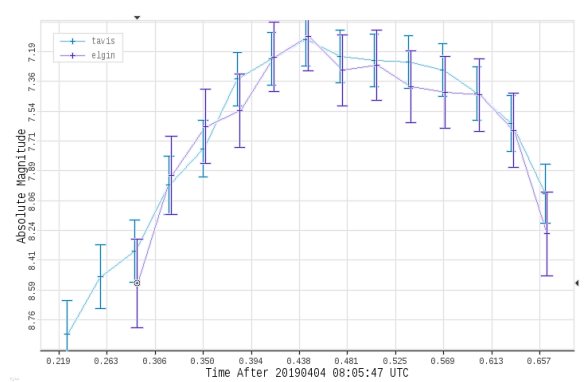

20190404:080547

20190404:040045

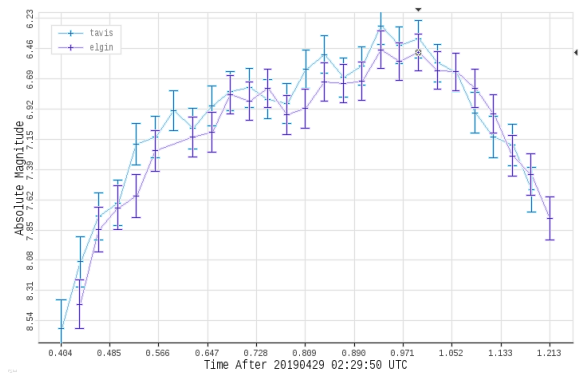

20190429:022950

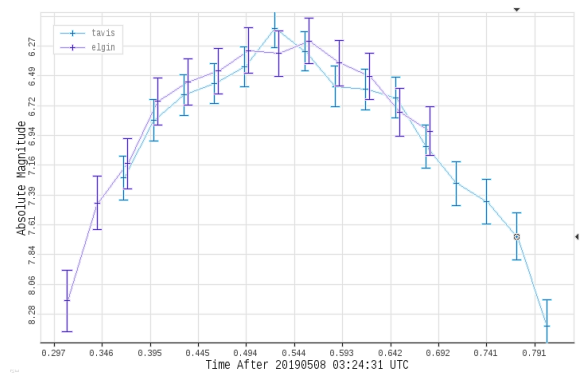

20190508:032431

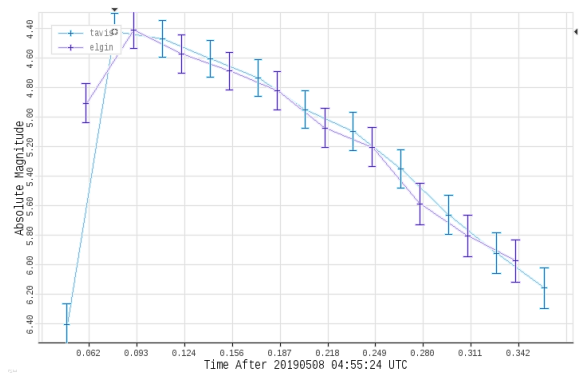

20190508:045524 


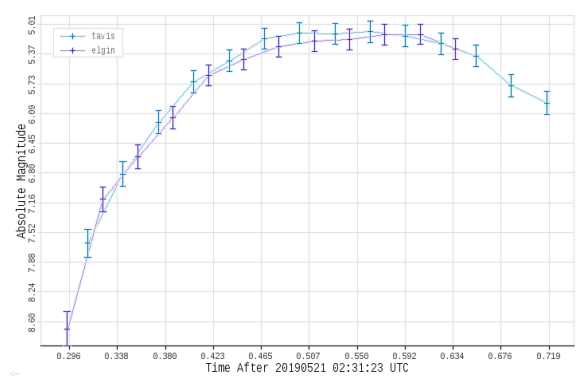

20190521:023123

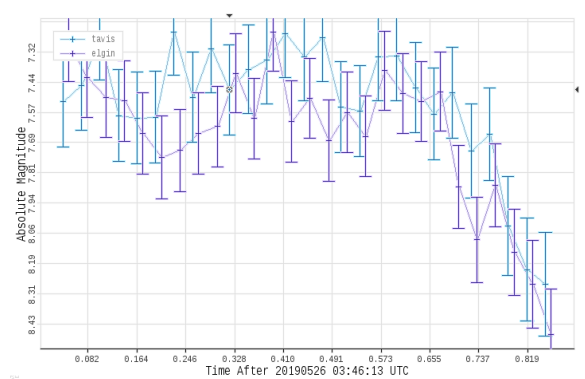

20190526:034613

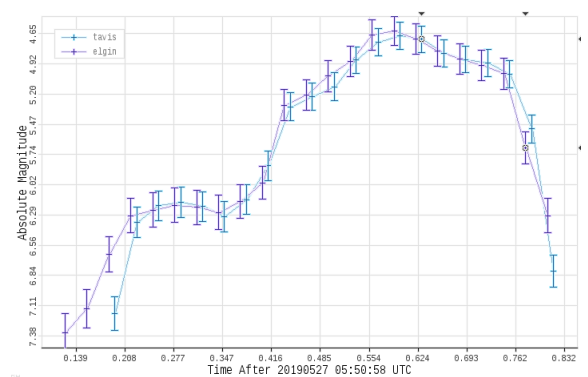

20190527:055058

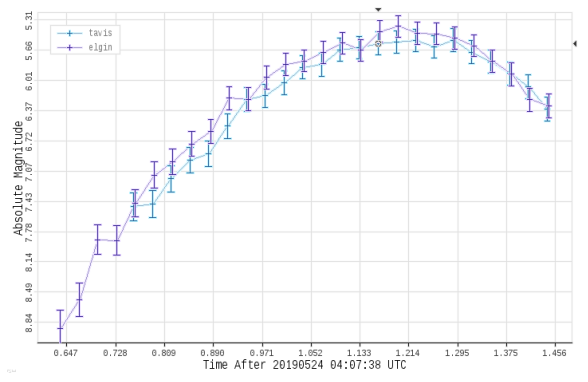

20190524:040738

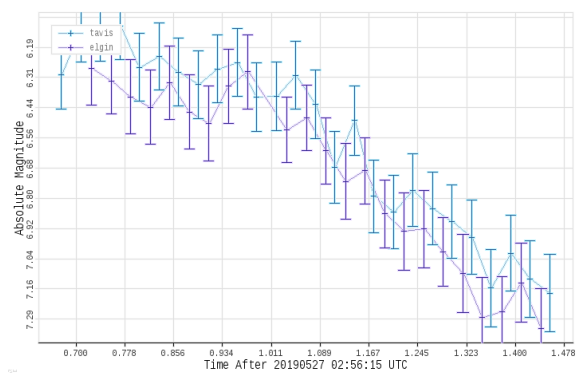

20190527:025615

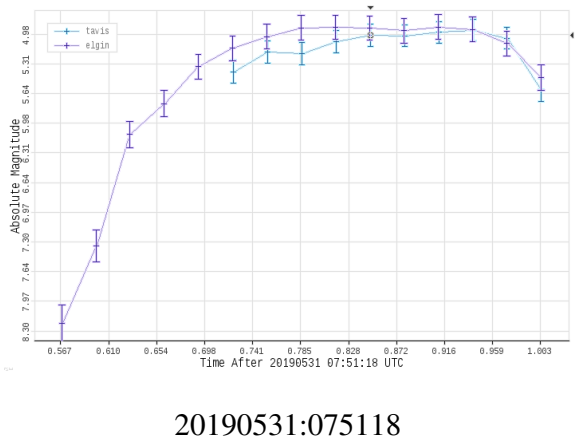

